\title{
TandEM: Titan and Enceladus mission
}

A. Coustenis - S. K. Atreya • T. Balint • R. H. Brown • M. K. Dougherty •

F. Ferri • M. Fulchignoni - D. Gautier • R. A. Gowen - C. A. Griffith •

L. I. Gurvits · R. Jaumann • Y. Langevin • M. R. Leese · J. I. Lunine •

C. P. McKay • X. Moussas • I. Müller-Wodarg • F. Neubauer • T. C. Owen •

F. Raulin • E. C. Sittler • F. Sohl • C. Sotin • G. Tobie • T. Tokano •

E. P. Turtle · J.-E. Wahlund · J. H. Waite · K. H. Baines • J. Blamont •

A. J. Coates · I. Dandouras · T. Krimigis • E. Lellouch • R. D. Lorenz •

A. Morse - C. C. Porco - M. Hirtzig • J. Saur • T. Spilker · J. C. Zarnecki •

E. Choi · N. Achilleos • R. Amils - P. Annan - D. H. Atkinson • Y. Bénilan •

C. Bertucci - B. Bézard · G. L. Bjoraker • M. Blanc · L. Boireau • J. Bouman •

M. Cabane - M. T. Capria - E. Chassefière · P. Coll • M. Combes •

J. F. Cooper - A. Coradini - F. Crary • T. Cravens - I. A. Daglis • E. de Angelis •

C. de Bergh • I. de Pater - C. Dunford • G. Durry • O. Dutuit • D. Fairbrother •

F. M. Flasar - A. D. Fortes • R. Frampton • M. Fujimoto • M. Galand •

O. Grasset • M. Grott • T. Haltigin - A. Herique · F. Hersant • H. Hussmann •

W. Ip • R. Johnson • E. Kallio • S. Kempf • M. Knapmeyer • W. Kofman •

R. Koop • T. Kostiuk • N. Krupp • M. Küppers • H. Lammer • L.-M. Lara •

P. Lavvas · S. Le Mouélic · S. Lebonnois · S. Ledvina • J. Li • T. A. Livengood

R. M. Lopes · J.-J. Lopez-Moreno • D. Luz • P. R. Mahaffy • U. Mall •

J. Martinez-Frias - B. Marty - T. McCord - C. Menor Salvan - A. Milillo •

D. G. Mitchell · R. Modolo • O. Mousis - M. Nakamura - C. D. Neish •

C. A. Nixon • D. Nna Mvondo • G. Orton • M. Paetzold • J. Pitman •

S. Pogrebenko • W. Pollard • O. Prieto-Ballesteros • P. Rannou • K. Reh •

L. Richter · F. T. Robb · R. Rodrigo • S. Rodriguez • P. Romani •

M. Ruiz Bermejo • E. T. Sarris • P. Schenk • B. Schmitt • N. Schmitz •

D. Schulze-Makuch • K. Schwingenschuh • A. Selig • B. Sicardy •

L. Soderblom · L. J. Spilker · D. Stam - A. Steele · K. Stephan · D. F. Strobel •

K. Szego • C. Szopa · R. Thissen • M. G. Tomasko • D. Toublanc • H. Vali •

I. Vardavas · V. Vuitton • R. A. West • R. Yelle $\cdot$ E. F. Young

Received: 14 December 2007 / Accepted: 27 May 2008 / Published online: 8 July 2008 (C) Springer Science + Business Media B.V. 2008

\footnotetext{
A. Coustenis $(\bowtie) \cdot$ M. Fulchignoni $\cdot$ D. Gautier $\cdot$ E. Lellouch $\cdot$ M. Hirtzig $\cdot$ B. Bézard · M. Combes · C. de Bergh · D. Luz · B. Sicardy

Laboratoire d'Etudes Spatiales et d'Instrumentation en Astrophysique (LESIA),

Observatoire de Paris-Meudon, 92195, Meudon Cedex, France

e-mail: athena.coustenis@obspm.fr
} 
Abstract TandEM was proposed as an L-class (large) mission in response to ESA's Cosmic Vision 2015-2025 Call, and accepted for further studies, with the goal of exploring Titan and Enceladus. The mission concept is to perform in situ investigations of two worlds tied together by location and properties, whose remarkable natures have been partly revealed by the ongoing CassiniHuygens mission. These bodies still hold mysteries requiring a complete exploration using a variety of vehicles and instruments. TandEM is an ambitious mission because its targets are two of the most exciting and challenging bodies in the Solar System. It is designed to build on but exceed the scientific and technological accomplishments of the Cassini-Huygens mission, exploring Titan and Enceladus in ways that are not currently possible (full close-up and in situ coverage over long periods of time). In the current mission architecture, TandEM proposes to deliver two medium-sized spacecraft to the Saturnian system. One spacecraft would be an orbiter with a large host of instruments which would perform several Enceladus flybys and deliver penetrators to its surface before going into a dedicated orbit around Titan alone, while the

S. K. Atreya $\cdot$ M. Hirtzig

Department of Atmospheric, Oceanic, and Space Sciences,

University of Michigan, Ann Arbor, USA

T. Balint · K. H. Baines · T. Spilker · R. M. Lopes · G. Orton · K. Reh ·

L. J. Spilker · R. A. West

JPL, Caltech, Pasadena, USA

R. H. Brown · C. A. Griffith · J. I. Lunine - P. Lavvas · C. D. Neish · M. G. Tomasko ·

V. Vuitton · R. Yelle

LPL, University of Arizona, Tucson, AZ, USA

M. K. Dougherty · I. Müller-Wodarg · C. Bertucci · C. Dunford · M. Galand

Imperial College, London, UK

F. Ferri

CISAS, Università di Padova, Padua, Italy

R. A. Gowen · A. J. Coates · N. Achilleos · A. D. Fortes

Mullard Space Science Laboratory, University College London, Bloomsbury, UK

L. I. Gurvits · S. Pogrebenko

Joint Institute for VLBI in Europe, Dwingeloo, The Netherlands

R. Jaumann · F. Sohl · M. Grott · H. Hussmann · M. Knapmeyer · L. Richter ·

N. Schmitz $\cdot$ K. Stephan

German Aerospace Center (DLR), Institute of Planetary Research, Berlin, Germany

Y. Langevin

IAS, Université Paris-Sud, Orsay, France

M. R. Leese - A. Morse - J. C. Zarnecki

Open University, Milton Keynes, UK

C. P. McKay

NASA/AMES, Palo Alto, CA, USA

Springer 
other spacecraft would carry the Titan in situ investigation components, i.e. a hot-air balloon (Montgolfière) and possibly several landing probes to be delivered through the atmosphere.

Keywords TandEM $\cdot$ Titan $\cdot$ Enceladus $\cdot$ Saturnian system $\cdot$ Landing probes

\section{Context}

TandEM was proposed in response to ESA's Cosmic Vision 2015-2025 Call. The proposal was constructed around an L-class mission to explore in situ Titan and Enceladus, two of the most interesting bodies in the Saturnian system, indeed in the Solar System. Titan's dense and optically thick atmosphere has been penetrated by the Cassini-Huygens mission (http://saturn.jpl.nasa. gov/mission/index.cfm) to reveal a complex world with diverse geophysical and

\footnotetext{
X. Moussas

Faculty of Physics, University of Athens, Athens, Greece

F. Neubauer $\cdot$ T. Tokano $\cdot$ J. Saur $\cdot$ M. Paetzold

University of Cologne, Cologne, Germany

T. C. Owen

Institute for Astronomy, University of Hawaii, Honolulu, USA

F. Raulin · Y. Bénilan · P. Coll

LISA, Universités Paris 12 and Paris 7, Créteil, France

E. C. Sittler · G. L. Bjoraker · J. F. Cooper · D. Fairbrother · F. M. Flasar · T. Kostiuk •

P. R. Mahaffy · C. A. Nixon · P. Romani

NASA/Goddard Space Flight Center, Greenbelt, MD, 20771, USA

C. Sotin · G. Tobie · O. Grasset · A. Herique · S. Le Mouélic

Lab. de Planétologie et de Géodynamique, Faculté des Sciences, Nantes, France

E. P. Turtle · T. Krimigis · R. D. Lorenz · D. G. Mitchell · D. F. Strobel

The Johns Hopkins University Applied Physics Laboratory, Laurel, MD, 20723, USA

J.-E. Wahlund · R. Modolo

Swedish Institute for Space Physics, Uppsala, Sweden

J. H. Waite · F. Crary · E. F. Young

Southwest Research Institute, San Antonio, USA

J. Blamont · L. Boireau

Centre National d'Etudes Spatiales, Paris, France

I. Dandouras $\cdot$ D. Toublanc

Centre d'Etude Spatiale des Rayonnements, Toulouse, France

T. Krimigis

Academy of Athens, Athens, Greece
} 
atmospheric processes reminiscent of those on Earth, but operating under very different conditions from our home world. Cassini and Huygens will leave us with an outstanding list of questions about Titan that are directly relevant to our understanding of the nature of planetary evolution, planetary processes, and planetary habitability. Cassini discovered that Enceladus is an active world with plumes spewing water, nitrogen and organic molecules into space; how such a tiny moon could be so active and whether the source of the activity is an organic-bearing liquid water region under the surface are questions that will require direct penetration through the surface for their answers.

The questions that will remain to be answered at the close of the CassiniHuygens mission have led this Consortium of Titan and Enceladus experts to propose a new mission to the Saturnian system, and to outline a technology development path for achieving Technology Readiness Level (TRL) 6 and for enhancing and enabling technologies so that such a mission can go forward in the Cosmic Vision timeframe. This mission concept addresses directly several

\section{C. Porco}

CICLOPS, SSI, Boulder, CO, USA

E. Choi

Bombardier Aerospace, Toronto, ON, Canada

R. Amils · J. Martinez-Frias · C. Menor Salvan · D. Nna Mvondo ·

O. Prieto-Ballesteros · M. Ruiz Bermejo

Centro de Astrobiologia, CSIC-INTA, Madrid, Spain

P. Annan

Sensors and Software, Mississauga, Canada

D. H. Atkinson

NASA, University of Idaho, Moscow, USA

M. Blanc

Ecole Polytechnique, Paris, France

J. Bouman $\cdot$ R. Koop $\cdot$ A. Selig $\cdot$ D. Stam

SRON, Netherlands Institute for Space Research, Utrecht, The Netherlands

M. Cabane · E. Chassefière · G. Durry · C. Szopa

Université Pierre et Marie Curie-Paris6, Service d'Aéronomie, Paris, France

M. T. Capria · A. Coradini · E. de Angelis · A. Milillo

Istituto Nazionale di Astrofisica, Rome, Italy

T. Cravens

University of Kansas, Lawrence, USA

I. A. Daglis

National Observatory of Athens, Athens, Greece

I. de Pater · S. Ledvina

Astronomy Department, University of California at Berkeley, Berkeley, USA 
of the scientific questions highlighted in the ESA Cosmic Vision 2015-2025 call, particularly: 1.3 "Life and habitability in the Solar System" and 2.2 "The giant planets and their environments", but also 2.1 "From the Sun to the edge of the Solar System".

TandEM is a multi-component exploration system to be built with international partners for a launch around 2018 or later. The mission concept has been developed through the expertise of 155 scientists and engineers from all over the world, many of whom are actively involved in the exploration of the Saturn system through Cassini-Huygens and/or have participated in mission designs for existing and future missions. Details on the mission can be found on http://www.lesia.obspm.fr/cosmicvision/tandem/index.php.

\section{Introduction}

Titan and Enceladus as revealed by the Cassini-Huygens mission are enigmatic objects, introducing extraordinary challenges for geologists,

O. Dutuit · W. Kofman · B. Schmitt · R. Thissen

Lab. Planétologie Grenoble, Univ. J. Fourier, CNRS, Grenoble, France

R. Frampton

Boeing, Chicago, USA

M. Fujimoto · M. Nakamura

Institute of Space and Astronautical Science,

Japan Aerospace Exploration Agency (JAXA),

Chôfu, Japan

T. Haltigin · W. Pollard · H. Vali

McGill University, Montreal, Canada

F. Hersant

Univ. de Bordeaux, Bordeaux, France

W. Ip

Institute of Astronomy, National Central University, Jhongli City, Taiwan

R. Johnson

University of Virginia, Charlottesville, USA

E. Kallio

Finnish Metereological Institute, Helsinki, Finland

S. Kempf · N. Krupp · M. Küppers · U. Mall

Max Planck Institute, Lindau, Germany

H. Lammer $\cdot$ K. Schwingenschuh

Space Research Institute, Graz, Austria

L.-M. Lara · J.-J. Lopez-Moreno $\cdot$ R. Rodrigo

Instituto de Astrofísica de Andalucía, CSIC, Granada, Spain 
astrobiologists, organic chemists, and planetologists; dreamworlds for those who yearn to explore mysterious new realms.

Cassini has shown that Enceladus possesses active plumes, the sources of which may be pockets of liquid water near the surface, an intriguing possibility for such a small and distant satellite. Little is known today about Enceladus, and after several flybys during Cassini's life we shall still lack precise knowledge on how this body's interior works or how it affects its surrounding environment (magnetosphere, rings, satellites), a serious drawback considering that we know that Enceladus is the primary source of mass loading of Saturn's magnetosphere (like Io for Jupiter).

Titan, on the other hand, Saturn's largest satellite, is unique in the Solar System with its extensive atmosphere made mostly of $\mathrm{N}_{2}$, with a column density ten times that of Earth's, and possessing a rich organic chemistry thanks to abundant methane. Titan's atmosphere is not in chemical equilibrium. It is a chemical factory initiating the formation of complex positive and negative ions

P. Lavvas · I. Vardavas

FORTH, University of Crete, Heraklion, Greece

S. Lebonnois

Labarotoire de Météorologie Dynamique, Paris, France

J. Li

LASG, IAP, Chinese Academy of Sciences, Taipei, China

T. A. Livengood

USRA National Center for Earth and Space Science Education, Columbia, USA

D. Luz

CAAUL-Observatório Astronómico de Lisboa, Tapada da Ajuda,

1349-018, Lisboa, Portugal

B. Marty

CRPG, Nançy, France

T. McCord

Bear Fight Center, Winthrop, WA, USA

O. Mousis

Institut UTINAM, Université de Franche-Comté, CNRS/INSU, Besançon, France

C. A. Nixon

Department of Astronomy, Univesity of Maryland, College Park, MD, 20742, USA

J. Pitman

Lockheed Martin Sensing and Exploration Systems, Denver, CO, USA

P. Rannou

Univ. Reims Champagne-Ardennes, Reims, France

F. T. Robb

University of Maryland Biotechnology Institute, Baltimore, MD, 21202, USA 
in the high thermosphere as a consequence of magnetospheric-ionosphericatmospheric interactions involving solar EUV, UV radiation, energetic ions and electrons. The second most abundant constituent, methane, is dissociated irreversibly to produce hydrocarbons (e.g. $\mathrm{C}_{2} \mathrm{H}_{6}$ and $\mathrm{C}_{2} \mathrm{H}_{2}$ ) and nitriles, (e.g. $\mathrm{HCN}$ ), from the coupled nitrogen chemistry. The energetic chemistry produces large molecules like benzene, naphthalene, etc., which begin to condense out at $\sim 950 \mathrm{~km}$, are detectable in solar and stellar UV occultations, and initiate the process of haze formation. As the haze particles fall through the atmosphere and grow, they become detectable with imaging systems such as the Cassini ISS at $\sim 500 \mathrm{~km}$ altitude and are ubiquitous throughout the stratosphere. They are strong absorbers of solar UV and visible radiation and play a fundamental role in heating Titan's stratosphere and driving wind systems in the middle atmosphere, much as ozone does in the Earth's middle atmosphere. Eventually, these complex organic molecules are deposited on Titan's surface in large quantities, where data from Cassini's instruments hint at their existence. Hence the upper thermosphere is linked intimately with the surface and the intervening atmosphere.

On Titan, methane can exist as a gas, liquid and solid. Playing a role similar to that of water on the Earth, methane is cycled between the atmosphere, surface and the interior. Cloud systems, the size of terrestrial hurricanes ( $\sim$ up to $1,000 \mathrm{~km}$ across), appear occasionally, while smaller transient systems form on a daily basis. The smaller clouds dissipate quickly suggesting the presence of precipitation, carving out the fluvial features that cover much of the equatorial landscape and are common also in the vicinity of lakes seen in the northern polar regions. Near-IR observations indicate that clouds exist mainly south of $60^{\circ} \mathrm{S}$, and in a band at $40^{\circ} \mathrm{S}$ latitude. Titan's cloud coverage appears to be less than that of the Earth, but highly variable. Titan's atmospheric methane may

S. Rodriguez

AIM/CEA, Saclay, France

E. T. Sarris

Demokritos University of Thrace, Xanthi, Greece

P. Schenk

Lunar and Planetary Institute, Houston, USA

D. Schulze-Makuch

School of Earth and Environmental Sciences,

Washington State University, Pullman, USA

L. Soderblom

US Geological Survey, Tucson, AZ, USA

A. Steele

Carnegie Inst, Washington, DC, USA

K. Szego

KFKI Research Institute for Particle and Nuclear Physics, Budapest, Hungary 
be supplemented by high latitude lakes and seas of methane and ethane [41], which over time cycle methane back into the atmosphere where it rains out, creating fluvial erosion over a wide range of latitudes.

After several close Cassini flybys, in January 2005, the Huygens Probe became the first human artifact to descend through Titan's atmosphere, reach the surface and return several hours of data from an exotic landscape cut by channels and apparently soaked, near the surface, with methane, ethane and other hydrocarbons (http://www.esa.int/SPECIALS/Cassini-Huygens/index. html). Several years of flybys by the Cassini orbiter have led to radar and near-IR maps that suggest surface liquids poleward of the $70^{\circ}$ latitude. Dunes made of frozen organic fine grains extend for thousands of kilometres. In the absence of a massive surface ocean, but with analogues to all other terrestrial hydrological phenomena present, Titan's methane cycle is indeed exotic.

The $\mathrm{N}_{2}-\mathrm{CH}_{4}$ byproducts in Titan's atmosphere eventually end up as sediments on the surface, where they accumulate presently at a rate of roughly $0.5 \mathrm{~km}$ in 4.5 Gyr. Since no large source was detected by Cassini to resupply methane, cryovolcanic outgassing has been hypothesized [42], yet over what timescales and through which internal processes are unknown. CassiniHuygens also found that the balance of geologic processes-impacts, tectonics, fluvial, aeolian - is somewhat similar to the Earth's, more so than for Venus or Mars. Titan may well be the best analogue to an active terrestrial planet in the sense of our home planet, albeit with different working materials.

The Cassini-Huygens mission is a remarkable success, answering many outstanding questions about the Saturn system and Titan in particular. As for many successful missions, the key contributions of Cassini may be the questions raised rather than those answered. An important limitation of Cassini, with respect to Titan science, is the insufficient spatial coverage allowed by its orbit around Saturn. While measurements have highlighted the complexity of Titan's atmosphere and magnetic environment, the coverage has been insufficient to achieve a full understanding. The minimum possible flyby altitude of $950 \mathrm{~km}$ and the uneven latitudinal coverage have limited our ability to explore the full set of atmospheric chemical processes. Opportunities for occultations have been rare, thus gaps remain in the atmospheric structure when in the magnetospheric downstream region. The single vertical profile of the atmosphere taken by Huygens limits our understanding of horizontal transport and latitudinal variations.

The surface of Titan, as revealed by the Huygens probe and the Cassini orbiter, offers us an opportunity to stretch our current models in an effort to explain the presence of dunes, rivers, lakes, cryovolcanoes and mountains in a world where the rocks are composed of water ice rather than silicates and the liquid is methane or ethane rather than liquid water, but the limited spatial coverage of high resolution imaging (corresponding to $25-30 \%$ with RADAR and much less with VIMS) limits our view of the range of coupled geologic, geochemical or energetic processes still going on Titan's surface and the interior. The exciting results from the Huygens post-landing measurements 
are limited to a fixed site, short timescales, and do not allow for direct subsurface access and sampling.

The two major themes in Titan exploration-the methane cycle as an analogue to the terrestrial hydrological cycle and the complex chemical transformations of organic molecules in the atmosphere and the surface-make Titan a very high priority if we are to understand how volatile-rich worlds evolve and how organic chemistry and planetary evolution interact on large spatial and temporal scales. Both are of keen interest to planetology and astrobiology.

The intriguing discoveries of geological activity, excess warmth and outgassing on Enceladus (due perhaps to the ejection of water and organics from subsurface pockets bathed in heat, or by some other mechanism), mandate a follow-up investigation to that tiny Saturnian world that can only be achieved with high resolution remote observations, and detailed in situ investigations of the near-surface south polar environment.

TandEM will investigate the science goals described in Section 3, with the mission scenario and the payload detailed in Sections 4, 5 and 6. Some outreach possibilities are given in Section 7.

\section{Scientific objectives}

The primary science goals of this mission are to understand Titan's and Enceladus' atmospheres, surfaces and interiors, to determine the pre- and proto-biotic chemistry that may be occurring on both objects, and to derive constraints on the satellites' origins and evolution, both individually and in the context of the complex Saturnian system as a whole (Table 1).

To achieve these goals, we need a mission that can visit both satellites and (a) study the atmosphere of Titan (including the "agnostosphere" a gap in Titan's atmosphere between 400 and $950 \mathrm{~km}$ altitude that will remain unexplored after Cassini), (b) image in detail, access, sample and analyze

Table 1 Characteristics of Titan and Enceladus

\begin{tabular}{lll}
\hline Characteristic & Titan & Enceladus \\
\hline Distance from Saturn & $20.25 \mathrm{R}_{\text {Sat }}$ & $3.95 \mathrm{R}_{\text {Sat }}$ \\
Period (days) & 15.95 & 1.37 \\
Orbit inclination $\left({ }^{\circ}\right)$ & 0.28 & 0.009 \\
Excentricity of orbit & 0.029 & 0.0047 \\
Mass $\left(10^{22} \mathrm{~kg}\right)$ & 13.5 & 0.011 \\
Radius $(\mathrm{km})$ & 2,575 & 252 \\
Density $\left(\mathrm{kg} / \mathrm{m}^{3}\right)$ & 1,880 & 1,606 \\
Gravity acceleration $\left(\mathrm{m} / \mathrm{s}^{2}\right)$ & 1.35 & 0.12 \\
Escape velocity $(\mathrm{km} / \mathrm{s})$ & 2.64 & 0.235 \\
Geometric albedo & 0.2 & 1.0 \\
Temperature at surface $(\mathrm{K})$ & 94 & $114-157$ \\
Pressure at surface $($ bar $)$ & 1.5 & $10^{-10}-10^{-13}$ \\
Main atmospheric components & $\mathrm{N}_{2}, \mathrm{CH}_{4}$ & $\mathrm{H}_{2} \mathrm{O}, \mathrm{N}_{2} / \mathrm{CO}, \mathrm{CH}_{4}, \mathrm{CO}_{2}$ \\
\hline
\end{tabular}


the various surface features (such as the hydrocarbon lakes, the dunes, river systems, impact craters, mountain ranges and volcanoes), aerosol deposits on the surface and any upwelled subsurface material, (c) determine whether Titan has a sub-surface liquid ocean, and, (d) investigate the atmosphere and penetrate or sample the surface of Enceladus.

In the following, we discuss the TandEM objectives for understanding Titan and Enceladus, their relationship and connection to the origin and evolution of the Solar System and their astrobiological implications.

\subsection{Titan as a system}

The Earth is studied as a complex system of coupled parts. And so is Titan. More than any other natural satellite in the Solar System, Titan exhibits somewhat similar coupling between magnetosphere, upper atmosphere and ionosphere, lower atmosphere, surface and the interior as the Earth. Indeed, because Titan possesses a cycle of condensation and evaporation of liquids from the surface, it is more like the Earth in this regard than present-day Venus or Mars.

The unifying working fluid in the terrestrial system is water. Water is present in the Earth's mantle and crust, modifying the rheology and chemistry of rock in profound ways. Plate tectonics recycles water in the crust back into the atmosphere and oceans. Water passes through liquid, solid and gas phases as a function of position and time, setting the basic climate state of the Earth through clouds and vapour and, via rainfall and ice, carving the landscape and altering climate. Water is photolyzed in the upper atmosphere, creating products that absorb and emit solar energy. Prior to the origin of life, Earth's atmospheric oxygen budget was driven by water photochemistry.

Cassini and Huygens investigations have suggested a role for methane on Titan equally profound as that of water on the Earth. Methane is likely present in the interior, dissolved in a liquid mantle and trapped in ice as clathrate hydrate, affecting the mechanical, thermal and chemical properties of the ice crust. Methane may be injected from the deep crust via volcanism and geysering, some of which could be explosive. These processes may be facilitated by the presence of a liquid water mantle under the crust. Surface (and potentially subsurface) reservoirs of liquid methane at high latitudes in the north, and likely in the south, affect the global methane cycle in ways that have yet to be fully understood. It is thought that on century to millennium timescales, convective downpours at mid-latitudes carve channels, erode the landscape, and move organic particulates into the lowlands. Methane in Titan's atmosphere forms clouds and helps drive the basic radiative balance of the dense atmosphere. High in the upper atmosphere of Titan, methane is broken apart by ultraviolet light and energetic particles and undergoes chemical processes, together with nitrogen, to form a host of complex organic molecules and dense aerosol layers. These compounds and aerosols fall to the surface where the liquids find their way into methane aquifers or into the polar 
lakes and seas, while the solid hydrocarbons and nitriles coat the surface and agglomerate into particles that are blown by the winds into vast fields of dunes.

This beautiful portrait of a planetary body as comprehensively active as the Earth, yet different in its working materials and details, is incomplete and still speculative. Cassini-Huygens has shown us direct and indirect evidence for many of the component parts. But confirmation of key players and how they fit together still eludes us.

We are motivated to ask the following key questions that we expect will require TandEM for their definitive answers, despite additional progress that Cassini and Huygens data may provide:

1. Are the "lakes and seas" filled with methane and ethane, and do they extend to a subcrustal hydrocarbon "methanofer" system over a larger area of Titan? Where is all the ethane? Are these processes affected by a deep water ocean, e.g. through fissures by tidal flexing?

2. What is the composition of the dune particles, and of the bright and dark materials on the surface?

3. Does Titan contain ammonia and to what extent has it been deposited on the surface?

4. What, if any, are the types of cryovolcanism on Titan?

5. What are the origins of the various mountain systems and, in general, what is the crustal history of Titan?

6. What are the magnitude and time variations of an internally generated magnetic field, if there is one, due to induction in an ocean or a weak dynamo?

7. What are the abundances of krypton and xenon, and hence what is the origin of Titan's methane?

8. What is the role of the essentially unexplored 'agnostosphere' in the chemistry and dynamics?

9. How is Titan's upper atmosphere coupled to the magnetosphere of Saturn?

10. What are the seasonal- and longer-scale dependencies of the distribution of methane among vapour, clouds and rain?

11. What are the temporal and spatial dependencies of the upper and lower atmospheric winds?

\subsubsection{Titan's upper atmosphere and magnetospheric interactions}

The proposed mission will investigate Titan's atmosphere, its ionosphere and exosphere as well as its plasma and magnetic field environment. Titan's upper atmosphere $(>400 \mathrm{~km})$ plays a key role for the chemistry and physics of the entire atmosphere. It is the region where solar EUV radiation is absorbed, dissociating molecular nitrogen and methane and initiating a chain of highly complex photochemistry that affects all layers. This is where Titan's atmosphere interacts with Saturn's magnetosphere. It is where Titan's induced magnetosphere forms and energy and particles from Saturn's magnetosphere 
are deposited, dissociating and ionizing nitrogen and methane, contributing to the complex atmospheric chemistry (http://saturn.jpl.nasa.gov/multimedia/ images/image-details.cfm?imageID=2176). Here, atmospheric and ionospheric escape processes also occur, directly affecting atmospheric evolution. The altitude range from $\sim 400$ to $950 \mathrm{~km}$ of the atmosphere is below the reach of Cassini in situ measurements but above the region of intense remote sensing and most Huygens measurements, and can justifiably be called the "agnostosphere".

Our knowledge of Titan's upper atmosphere and magnetospheric interaction is due to Voyager (1980), Earth-based and the recent Cassini/Huygens observations, which have given us an unprecedented insight into Titan's complex upper atmospheric chemistry and its relationship to the magnetosphere. However, Cassini can only sample the atmosphere in situ down to $950 \mathrm{~km}$ altitude, while Huygens obtained a single vertical profile of total atmospheric density from below $1,400 \mathrm{~km}$ and a much more detailed set of measurements below $\sim 140 \mathrm{~km}$ to the surface. Further information on Titan's upper atmosphere was obtained from ground-based and Cassini observations of stellar occultations. The major recent discoveries in Titan's upper atmosphere include its chemical complexity ( $\sim 100$ inferred organic molecules), the presence of haze at high altitudes which are at different altitudes from those at which Voyager observed haze layers, strong horizontal variations, vigorous dynamics and intimate coupling of the ionosphere to the magnetic field. The observed heavy negative ions $(\sim 5,000 \mathrm{amu})$ in Titan's ionosphere [7] indicate that the ionosphere may provide the seed population for aerosol formation deeper down in the atmosphere. For the exosphere, neutral gas escape rates have been found to be much larger than previously assumed. A neutral hydrogen cloud was detected, extending out to many Titan radii. Observations of energetic neutral atoms (ENA) have been used to study magnetosphere dynamics near Titan. New results have been obtained regarding the incident magnetospheric flow conditions at Titan, the plasma composition outside and inside Titan's induced magnetosphere and the magnetotail-wake.

The prime objectives for the upper atmosphere/magnetosphere science by TandEM are:

1. Investigate the structure, chemistry, dynamics and energy balance of the largely unexplored agnostosphere between about 400 and $950 \mathrm{~km}$ and investigate the coupling of dynamics and chemistry. Understanding this broad transition region is a key component in understanding the entire atmosphere together with its relevance for astrobiology.

2. Investigate in detail the chemistry of Titan's upper neutral atmosphere and ionosphere, the formation of heavy hydrocarbons, positive and negative ions and aerosols due to ionization and dissociation by EUV and energetic particle radiation. Cassini instruments are not capable of detecting species at high mass and at the accuracy needed. A key suite of measurements involve the precipitated aerosols, starting with their composition, including isotopic ratios. This will require a clever GCMS outfitted with getters 
that can remove various species and concentrate others. It must have the capability of reaching high atomic weights-beyond $150 \mathrm{amu}$.

3. Analyze the horizontal structure and dynamics of Titan's upper atmosphere and investigate the effects on the chemistry.

4. Investigate the induced magnetosphere of Titan with its unique incident flow conditions. Study the magnetoplasma environment at different positions of Titan around Saturn.

5. Investigate the coupling of Titan's upper atmosphere to Saturn's magnetosphere. Study correlation between the plasma and neutrals in Titan's corona in order to reveal the mechanisms for atmospheric escape of both neutrals and ion.

6. Derive the characteristics of a possible internal magnetic field and evaluate the implications in the context of other large icy satellites, e.g., Jupiter's satellite Ganymede, which has a magnetic field, and Callisto which does not.

These goals are important contributions to themes 2.2 but also 2.1 of ESA's Cosmic Vision Plan. Our improved understanding of bodies within our Solar System can also be applied to exo-planets.

To achieve these objectives measurements described in Section 5.1 will be carried out. A dedicated Titan orbiter is essential to accomplish these tasks, and some of the required measurements prefer a spinning orbiter element.

\subsubsection{Titan's neutral atmosphere}

One overarching goal of the TandEM mission is to understand the workings of Titan's atmosphere, which, similar to Earth, is coupled to the interior (e.g. through outgassing), the surface (e.g. through evaporation and precipitation), and the ionosphere (e.g. through the chemistry).

A fundamental outstanding question about Titan pertains to its circulation, and the role played by the heating and cooling of the globally varying composition of Titan's atmosphere. Titan's temperature profile follows similar trends to that of Earth. At the surface, both atmospheres are warmed by greenhouse gases $(\sim 20 \mathrm{~K}$ for Titan and $\sim 35 \mathrm{~K}$ for Earth), mainly water $(30 \mathrm{~K})$ and $\mathrm{CO}_{2}$ on Earth, and pressure-induced $\mathrm{N}_{2}, \mathrm{H}_{2}$ and $\mathrm{CH}_{4}$ absorption on Titan. The temperature decreases with altitude from the surface up to the tropopause ( $\sim 10-15 \mathrm{~km}$ on Earth and $\sim 40 \mathrm{~km}$ on Titan). Above the tropopause, the atmosphere heats up due to ozone on Earth and to a balance between warming by haze and methane, and cooling due mainly to haze, $\mathrm{C}_{2} \mathrm{H}_{2}, \mathrm{C}_{2} \mathrm{H}_{6}$ and $\mathrm{HCN}$ on Titan. Here the atmospheres differ significantly in structure. Titan's haze, which enshrouds the moon and limits remote-sensing investigations of Titan's lower atmosphere and surface, absorbs $\sim 30 \%$ of the incoming solar radiation, acting like an "anti-greenhouse gas", and diminishing the illumination of the lower atmosphere [25]. Furthermore, the heating and cooling of the atmosphere by haze and other photolysis by-products $\left(\right.$ e.g. $\mathrm{C}_{2} \mathrm{H}_{2}$, $\mathrm{C}_{2} \mathrm{H}_{6}$ and $\mathrm{HCN}$ ) are complicated because their abundances differ dramatically 
with latitude and season [8], due to their entrainment in seasonal winds. Titan's atmospheric circulation affects the distribution of gases and haze, and their condensation and deposition on Titan's surface. In parallel, resultant latitudinal abundance variations affect the circulation. To understand Titan's circulation, seasonal measurements of the stratospheric composition are needed, which Cassini will sample only from roughly southern summer solstice to northern spring equinox. At the close of the Cassini mission, we will still not know unambiguously the haze densities in winter and summer polar regions, which differ, perhaps by an order of magnitude, from equatorial values.

Another intriguing question concerns the origin of winds in the upper stratosphere which rotate much faster than the surface, coupled with an unexpected layer of low wind speeds in the lower stratosphere as well as several reversals of the wind direction detected by Huygens near the surface. Elucidating the details of the transport pattern of atmospheric angular momentum is crucial in understanding the mechanism by which super-rotation is maintained. To this purpose, measurements of wind profiles at different places and seasons and a better characterisation of the nature of eddies, waves and tides are needed.

Titan's methane cycle, the source of methane and the processes that govern its surface and atmospheric distribution, are poorly understood. Despite the irreversible attrition of methane, Huygens measured a largely methane-saturated troposphere [49]. The methane inventory on Titan differs significantly from that of water on Earth. Titan's atmosphere has $\sim 5 \mathrm{~m}$ of precipitable methane, whereas Earth's has $2.5 \mathrm{~cm}$ of precipitable water vapour. Titan's known lakes and seas cover $\sim 1 \%$ of the surface, whereas oceans cover $70 \%$ of the Earth. It is thus extraordinary that Titan's lower atmosphere holds as much methane as it does, with a $45 \%$ relative humidity in an equatorial environment that geologically is dominated by dunes. While the polar lakes and seas might contain enough methane to periodically saturate the atmosphere, allowing violent rainstorms to carve river channels, they cannot be the ultimate source of the methane.

Perhaps volcanism in recent times has released just enough methane to saturate the atmosphere and leave the surface fairly dry. Or perhaps there is a steady-state source communicating to the surface in a way that has not been identified. Such questions require detailed measurements of the tropospheric methane content (globally) and the composition of the surface. Long radiative and dynamical timescales in the lower atmosphere make answering these questions with a single mission difficult, and hence a return to Titan imperative.

Another open question pertains to the formation of Titan's clouds. On Titan, only $10 \%$ of incident sunlight, compared to Earth's $60 \%$, reaches Titan's surface to drive weather. Despite the fact that the radiative time constant ( 140 Earth years) exceeds Titan's year (29.5 Earth years), large convective storms are observed to develop in Titan's atmosphere, apparently varying with season [13]. Images of clouds from Earth-based observatories and by Cassini, indicate a peculiar and unexpected pattern: over the approximate decade of observations, clouds have appeared most regularly south of $60^{\circ} \mathrm{S}$ latitude, 
in the North polar region (http://saturn.jpl.nasa.gov/multimedia/images/ image-details.cfm?imageID=2470 ) and in a band around $40^{\circ} \mathrm{S}$ latitude. Various theories have been proposed to explain their formation, involving atmospheric circulation, surface heating and cryo-volcanism. Yet these models cannot be tested because the structure of the atmosphere (e.g. humidity, winds and temperature profile) and the surface conditions (e.g. its dampness, temperature and evaporation rates) near storms will not be measured by Cassini. In particular, in situ measurements are needed at high latitudes (where clouds and lakes reside) to further understand Titan's atmospheric engine.

Titan's neutral atmosphere parallels Earth's, with a complex meteorology that cycles methane from the surface to the atmosphere, and a circulation pattern and atmospheric dynamics that affects the surface composition and geology (Fig. 1). Cassini, while it has provided the first glimpses into the complexity of the surface and lower atmosphere, will not obtain significant information on the surface humidity, the temperature profile and its variations in the boundary layer (near the surface), the atmospheric winds, the composition of the surface, and the source of atmospheric methane. In addition the seasonal structure (composition and temperature) will be sampled only from northern winter to spring. TandEM will address these omissions by (a) sampling a different season from Cassini or Voyager, (b) remaining operational across a significant fraction of a Saturnian (Titanian) year.

The prime objectives for the lower atmospheric science by TandEM are:

1. Determine the near surface temperature and temperature profile in the polar troposphere.

2. Search for evidence of atmospheric tides in the thermal profile.

3. Map out the meridional circulation of the troposphere, and its change with seasons.

4. Seek evidence for orographic winds and clouds, and winds associated with tropospheric convective clouds.

Fig. 1 Main couplings between Titan's atmospheric processes

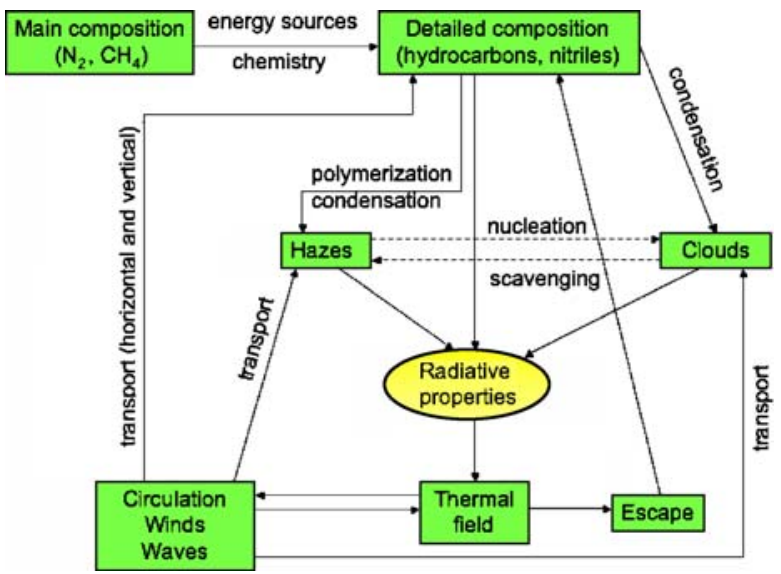


5. Characterise the structure and seasonal evolution of the polar vortex and its relevance for Titan's meteorology.

6. Map the seasonal and latitudinal variation in tropospheric methane abundance.

7. Determine the time-dependent physical and chemical properties of tropospheric methane/nitrogen clouds, and possible ethane ground fog (including electrical properties).

8. Search for evidence of methane outgassing on the surface, and methane evaporation from lakes.

9. Determine the nature of polar winter tropospheric cloud and precipitation.

10. Quantify the coupling of the surface and atmosphere in terms of mass and energy balance. Do further chemical reactions among aerosols and atmosphere take place on the surface? In the lakes/seas? On shorelines? In playas? Is their any surface segregation of these precipitated aerosols, driven by aeolian or fluvial processes?

The objectives that we delineate point to the set of measurements described in Section 5.1.

\subsubsection{Titan's surface}

The combination of geomorphologic information from high-resolution RADAR coverage (few hundred meters/pixel: http://saturn.jpl.nasa.gov/ multimedia/images/image-details.cfm?imageID=1176) with spectroscopic information from VIMS at more moderate resolution (few hundred meters/pixel to a few kilometres/pixel) have addressed the first order geological and chemical processes in a limited geographical area on Titan's surface. RADAR and VIMS together with Huygens high-resolution imaging and in situ studies have completed a preliminary survey of the chemical and physical processes in an area of order $100 \mathrm{~km}^{2}$. Combined ISS, VIMS and RADAR data [38] have provided near-global surface coverage at a variety of wavelengths and resolutions (http://saturn.jpl.nasa.gov/multimedia/images/imagedetails.cfm?imageID=2385).

Together, Cassini and Huygens have established a diversity of geologic features comparable to that on the Earth:

- erosional features such as channels and dendritic networks (http://saturn. jpl.nasa.gov/multimedia/images/image-details.cfm?imageID=1326 ), possible lakes and seas, fluvial erosional deltas and other erosional and depositional constructs, possible glacial-flow constructs, as well as widespread aeolian, erosional and depositional features such as dunes: (http://saturn. jpl.nasa.gov/multimedia/images/image-details.cfm?imageID=2260);

- impacts: the very low crater frequency [21] is indicative of active geological surface processes; 
- volcano-tectonic features (http://saturn.jpl.nasa.gov/multimedia/images/ image-details.cfm?imageID=1558): domes, possible cryovolcanic flows, and bright spots as well as mountain chains (http://saturn.jpl.nasa.gov/ multimedia/images/image-details.cfm?imageID=2381).

However, detailed knowledge of the global distribution of these features, their possible associations and causal relationships, their ages and the geophysical processes that are responsible for their origin and evolution remain poorly understood, even where Huygens landed [39], http://saturn.jpl.nasa.gov/ multimedia/images/image-details.cfm?imageID =1881, http://saturn.jpl.nasa. gov/multimedia/images/image-details.cfm?image ID $=1310$.

The deposition of $\mathrm{CH}_{4}-\mathrm{N}_{2}$ photochemical materials, including liquid ethane, suggest that Titan's surface will have lakes and seas of liquid hydrocarbons, which may have been found already in the high latitude regions, making a uniquely evocative landscape. At high spatial resolution Titan's landscape may be quite exotic, because the working materials differ so much from those on the Earth.

The prime objectives remaining for the surface and interior science with TandEM are:

1. Determine the ages and temporal relationships of various geologic units and constructs.

2. Seek the extent, timescale and mechanisms of volcanism and tectonism.

3. Determine the nature of erosion on Titan, its spatial extent and the relative balance of aeolian, fluvial, chemical, and glacial processes. Are there active fluvial channels, carrying liquid and eroding the surface?

4. Determine Titan's interior structure to a level of accuracy and confidence higher than that of Cassini, to assess the size of any internal ocean, core, and extent of differentiation.

5. Determine the age of the surface, the structure of the crust and mantle, and how the interior has interacted with the surface and atmosphere over time.

6. Determine the composition, distribution and physical state of materials on and beneath Titan's surface and how are they related to geology. Search for ammonia as an indicator of volcanism, and the presence of a liquid water-ammonia mantle. Does Titan still contain ammonia in its ice? If yes, does it reach the surface by itself or mixed with ice? if so, what is its lifetime? Seek evidence of sulfur, carbon dioxide, and other constituents, key to understanding Titan's origin and evolution.

7. Determine the source of the atmospheric methane, its outgassing history, and the surface/interior sinks for ethane and other photochemical products.

8. Determine the origin of Titan's major volatiles, such as methane and nitrogen, and structural materials such as ice and rock through noble gas abundances and isotopic ratios of hydrogen (D/H in $\mathrm{H}_{2} \mathrm{O}$-ice), carbon and nitrogen in abundant organic and inorganic material, sulfur (if present), and at a level much more sensitive than provided by Cassini/Huygens. 
TandEM will have the requisite capabilities to make the measurements described in Section 5.1 that are required to address these fundamental objectives

\subsection{Enceladus as a system}

The discovery by the Cassini spacecraft of a large plume emanating from surface cracks near the south pole of Enceladus [9] venting water vapour (http:// saturn.jpl.nasa.gov/multimedia/images/image-details.cfm? imageID=1629), fine icy particles (or dust) and organics implies an underground heat source. Whether the plumes emissions arise directly from chambers of pressurized liquid water, or evaporation from warm ice, or the explosive release of gases from clathrate ices is unknown. The origin and time history of the internal heating source is a major puzzle. Ions formed from the emissions are driven out into the magnetosphere of Saturn, and are implanted into the $\mathrm{E}$ ring grains, the other icy satellites, and even into Titan's atmosphere. Fine icy particles escaping from the jets (http://saturn.jpl.nasa.gov/ multimedia/images/image-details.cfm?imageID=2027) also drift outward forming the E-ringmake their way into the ring; confirming Enceladus as the main source for this ring. Cassini observations (http://saturn.jpl.nasa.gov/ multimedia/images/image-details.cfm?imageID=2779), including extended mission observations, will allow direct plume sampling as well as further remote sensing observations of the south polar regions and high phase plume imaging. With the TandEM mission we plan to extend both remote sensing and in situ measurements beyond Cassini capabilities, with more advanced instrumentation. We will remotely monitor the satellite and its activity over time, at high spatial resolution and over a range of different wavelengths and also obtain in situ measurements via plume fly-throughs. We also plan to deploy penetrators to the south polar terrain for making direct measurements of particular regions in the geologically active zone.

Our primary science objectives include:

- Origin, nature and properties of the plume (including particle dynamics, temporal variability, spatial distribution and production rates of gas/dust, association with local hot spots, etc.)

- Existence, depth, extent and composition of sub-surface liquid water (including degree of tidal flexing in the liquid zone, vent structure, liquidwater/clathrates ratios, overall composition, see Section 3.3.2)

- Signs of past/present life (including organic inventory, molecular chirality, etc. see Section 3.4)

- Some secondary objectives would be to:

- Characterization of the surface (including resurfacing processes, geometry of fractures, crater morphology, see Section 3.2.2)

- Characterization of the interior (including structure and mass distribution, gravity field, global topography, endogenic and exogenic dynamics, see Section 3.3.2) 
- The impact of Enceladus on the magnetosphere (including magnetospheric processes, plasma loading effects)

In the process of addressing the above objectives we would also discover answers to important unresolved questions such as the:

- Influence of Enceladus on other satellites

- Influence of Enceladus on the ring structure

- Determination of dust flux into system

Some of the detailed science questions linked to these priorities to be addressed by TandEM are given below, some are addressed in Sections 3.3 and 3.4, and the requisite measurements are given in Section 5.2.

\subsubsection{Nature of the plume source, variability and composition}

Single point measurements from the only close Cassini flyby of Enceladus to date have allowed us to gain a basic understanding of the composition of the plume, incorporating water vapour, dust and organics. In order to ascertain the variability of the plume, which has been suggested by the Cassini magnetometer and energetic particle observations, numerous fly-throughs of the plume separated both temporally and spatially as well as visual temporal monitoring of the plume over a range of phase angles and distances will be required. Understanding specific properties of the plume, its variability and its composition will lead to an understanding of the nature of its source.

Essential objectives include exploration of the:

- Surface distribution of temperatures

- Radiation environment

- Dynamic and physical properties of the plume 'dust' particles

- Spatial distribution of the gas and dust and its temporal variability

- Gas phase composition; noble gas determination, gas/dust determination

- Gas and particle production as function of time

- Pick-up ion production from solar UV and magnetospheric irradiation

\subsubsection{Characterise the surface of Enceladus}

Although Cassini/ISS has produced superb images of Enceladus' surface (http://saturn.jpl.nasa.gov/multimedia/images/image-details.cfm? imageID = 2032), at present, large regions of the surface of Enceladus have still not been imaged at a resolution sufficient to determine precise geological relationships and geologic history in detail. In order to be able to characterize large-scale, small-scale, and dynamic geological features on the surface we require repeated coverage at a variety of latitudes and longitudes at high resolution. Coverage spanning a variety of temporal scales-from hours, to days, weeks, months and even years-would be especially important for evaluating models for the geologically active south polar region. 
Specific science objectives related to the characterization of surface features include:

- Resurfacing processes; viscous relaxation; tectonic overprinting; deposition from plumes

- Surface ages and composition

- Vent structure, composition, temperature in the south polar region

- Craters: inventory, morphology, terrain dating, thermal gradient, spatial variability

- Magnetospheric irradiation environment

- Biological potential: organic inventory, molecular chirality, carbon and oxygen isotropic ratios

\subsubsection{The impact of Enceladus on its environment}

We will investigate:

- How the structure of the E ring is driven by and dependent on plume and outgassing processes from Enceladus.

- The magnetospheric processes. Measurements of the composition arrival directions and energy distribution of neutral atoms will yield information about internal composition, erosion of the upper surface, emission rate and the interaction with the magnetosphere.

- How does Enceladus populate Saturn's magnetosphere with neutrals, plasma and dust particles.

- The influence on other satellites via implantation of ions to their surfaces and driving many of the processes of Saturn's magnetosphere and its interactions with the other icy satellites. It may be the dominant source of water and oxygen delivered to Titan's atmosphere.

The full description of the measurements to be performed in order to achieve the science goals presented here are given in Section 5.2.

\subsection{Origin, interior and evolution}

The dense nitrogen-methane atmosphere of Titan and the spectacular waterrich, gas plumes of Enceladus offer some key challenges: Why should Saturn host such volatile and active worlds? How did they form? What internal processes make Titan and Enceladus so different from the other icy moons?

The fact that Titan has an atmosphere, something that the Galilean satellites do not possess, as well as the Enceladus ejecta furthermore suggest that the former is a sort of end-point for satellite formation around a giant planet, while the latter is a body from which primordial satellite materials are still being emitted. These key elements give us the opportunity to enhance our understanding of ours and other planetary systems (even satellites around giant exoplanets). The Cassini-Huygens mission has started to answer these questions, but key measurements are still missing to draw a complete picture 
of Titan's and Enceladus' origin and evolution. In the following sections, we detail the key objectives and measurements to be acquired in the frame of the TandEM mission to address those questions.

\subsubsection{Origin of Titan and Enceladus}

Connecting a model for the solar nebula to a model of giant planet formation that includes the formation of regular satellite systems is quite a difficult problem. Using a 3-D hydro-dynamical model, [22] showed that in the last stage of giant planet formation, a protosatellitary disk of captured material gradually emerges from the contracting atmosphere. Satellite formation could have taken place in this disk. Any successful model for satellite formation must include predictions for the variety of satellite densities and the probable compositions of atmospheres and surfaces. These are fundamental properties that can be deduced from in situ and orbital observations to better constrain satellite formation models. Unfortunately, critical observations of key cosmochemical elements required for the discrimination between competing formation models of Titan and Enceladus are rare or even lacking.

Titan We are far from understanding the origin of Titan. Scenarios may be envisaged for the origin of the planetesimals that formed Titan, based on two end-member type hypotheses:

1. Planetesimals were produced early, just after or even before the end of the formation of Saturn, within a relatively warm subnebula. In this case, only silicates and moderately refractory volatiles could have been incorporated in the planetesimals that formed Titan and the other indigenous regular satellites. It is then difficult to trap the noble gases and $\mathrm{CH}_{4}$ in icy grains but the trapping of $\mathrm{H}_{2} \mathrm{O}, \mathrm{NH}_{3}, \mathrm{CO}_{2}$ and non-volatile compounds would be facilitated $[1,5,30,31]$. The small amount of ${ }^{36} \mathrm{Ar}$ detected by the Huygens GCMS $\left({ }^{36} \mathrm{Ar} / \mathrm{N}<1 / 1000 \times\right.$ Earth's ratio, [27]) implies an ambient formation temperature of $\sim 100 \mathrm{~K}$ in the sub-nebula [30,31], under the assumption that Argon was trapped in amorphous ice. It has been proposed that methane on Titan was not initially incorporated, but was produced within the satellite from chemical (catalysed) reactions between carbon compounds such as $\mathrm{CO}_{2}$ and $\mathrm{H}$ produced from $\mathrm{H}_{2} \mathrm{O}$ by water-rock reactions [5].

2. Alternatively, the primary sub-nebula may have dissipated rapidly (possibly due to turbulence). The planetesimals were then produced in the cold, gas-dominated solar nebula near the end of its existence [16]. This is consistent with observations of circumstellar disks around young stars showing that their temperature dramatically decreases with time in no more than $10 \mathrm{Myr}$. Like in the former scenario, $\mathrm{NH}_{3}$ and $\mathrm{CO}_{2}$ condensed, while $\mathrm{CH}_{4}, \mathrm{H}_{2} \mathrm{~S}$ and $\mathrm{Xe}$ were trapped in the form of clathrate hydrate, being incorporated into in the migrating planetesimals that eventually formed Titan $[15,16]$. The deficiency in water ice at late epochs severely 
limited the trapping of less stable species such as $\mathrm{CO}$ and $\mathrm{Ar}$, clathrated at low temperatures (around $30 \mathrm{~K}$ ), much later than $\mathrm{CH}_{4}$, thus explaining their low abundances in Titan's atmosphere. In this scenario, the nondetection of Xe by the Huygens GCMS would be explained by preferential sequestration within Titan's crust and deep interior [16].

In both of these hypotheses, $\mathrm{NH}_{3}$ was released from the planetesimals during accretion, forming the early atmosphere. Subsequently, the conversion of $\mathrm{NH}_{3}$ into $\mathrm{N}_{2}$ may have been caused by photolysis in Titan's hot protoatmosphere [4] or impact-induced high temperature chemical processes [24], whereas $\mathrm{CH}_{4}$ should have been outgassed from the interior, as is ${ }^{40} \mathrm{Ar}[27,47]$. Although the process of atmospheric formation would be similar in the two scenarios, the resultant deuterium-to-hydrogen $(\mathrm{D} / \mathrm{H})$ ratio in vaporized water ice and methane should be different. The detection of $\mathrm{Kr}$ and $\mathrm{Xe}$ or sulfur compounds on the surface, which could not be achieved by the Huygens probe, will help discriminate between these two hypotheses.

Enceladus A key question is whether Enceladus formed from primordial bricks similar to those of Titan. In contrast to the Jovian system, which is characterized by a negative gradient in the satellite uncompressed density with distance from Jupiter, the Saturnian system exhibits large variations in mean density and mass of the satellites. So we should expect great variability also in the satellites' bulk chemical composition. The INMS instrument on board Cassini has found non-condensible volatile species (e.g. $\mathrm{N}_{2}, \mathrm{CO}_{2}, \mathrm{CH}_{4}$ ) in jet-like plumes over Enceladus' geologically active South Polar Terrain [50]. Some species, like for instance $\mathrm{CO}_{2}$, might be primordial, whereas others, like $\mathrm{N}_{2}$, might require a liquid water reservoir in the interior at elevated temperatures, enabling aqueous, catalytic chemical reactions [23]. Taken together, the evidence strongly suggests that Enceladus' interior is at least partially differentiated into a silicate-metal core overlain by a water ice-liquid water shell [37]. However, the energy source required to initiate and preserve activity at the level comparable to that observed today over geologic periods and the concentration of geologic and thermal activity towards the south-polar region are still not well understood.

Determining the composition of Enceladus' plumes will provide strong constraints on the origin of volatiles on Enceladus, and more generally in the entire Saturnian system. Even though the two satellites formed from satellitesimals with slightly different compositions, the detection of $\mathrm{CO}_{2}$ emanating from Enceladus' interior suggests that $\mathrm{CO}_{2}$ may also be present within Titan's interior. Comparison between Enceladus and Titan's composition will help constrain their origin and also bring some insight on the origin of the Galilean satellites.

Measurements that are required to better constrain the formation scenario of Titan and Enceladus are delineated in Section 5.3.1. A key measurement in this is the value of $D / H$ in Titan's ice. It can tell us the source of the ice, meaning the process(es) that formed it, hence conditions in Saturn's subnebula. It will 
also help us determine the source of Titan's methane-whether captured by ice forming in the subnebula, the solar nebula, cometary impacts, or produced in situ.

\subsubsection{Interior and early evolution}

Many internal processes play crucial roles in the evolution of Titan and Enceladus. The formation and replenishing of Titan's atmosphere and the jet activity at Enceladus' South Pole are intimately linked to the satellite's interior structures and dynamics. The primary objectives of the TandEM mission regarding Titan and Enceladus' interiors will be (1) to determine their presentday structures and levels of activity (2) to determine whether the satellites underwent significant tidal deformation, and whether they possess intrinsic or induced magnetic fields and significant seismicity, (3) to identify heat sources, internal reservoirs of volatiles (in particular methane and ammonia) and eruptive processes.

Present-day interior structure: rocky core and liquid water/ice shells The Radio Science Subsystem on board Cassini, by measuring the principal components of Titan's and Enceladus gravitational potential [34,35], will provide important constraints on the satellites' internal differentiation. However, the sizes of their cores and the thicknesses of their ice mantles will remain uncertain [40]. Determining the present-day structure of the outer ice mantle and of the innermost core is crucial to determining how a satellite's interior has differentiated over time and the effects on the evolution of the surface and atmosphere (http://www.sciences.univ-nantes.fr/geol/UMR6112/Nature\% 20Titan\%20outgassing_short_english.htm).

Therefore, the next step will be to characterize the sizes and states of the rock-metal cores, and the structure of the outer $\mathrm{H}_{2} \mathrm{O}$ layers. On Titan, joint measurements of large-scale and meso-scale topography and gravitational field anomalies from an orbiter and from an aerial platform would impose important constraints on the thickness of the lithosphere, the presence of mass anomalies at depth and any lateral variation of the ice mantle thickness. On Enceladus, altimetric and gravimetric profiles performed during close flybys $(<100 \mathrm{~km})$ over the South Pole will be used to retrieve the internal structure below the active region (depth of liquid reservoirs, lithosphere thickness, existence of thermal plumes in the ice mantle, in the rocky core) (e.g. [32]). These data will provide fundamental constraints on the origin of the South Pole Hotspot on Enceladus.

Tidally-induced deformation, magnetic field and seismicity The Radio Science measurements performed by Cassini at Titan should be a priori capable of detecting any tidal fluctuations of the gravitational potential [34]. The detection of amplified periodic signals would suggest the existence of a liquid layer at depth, most likely composed of liquid water [40,46]. However, even if these tidal fluctuations were detected, they would not provide firm constraints 
on important structural parameters such as the depth and radial extent of the liquid water reservoir, the thickness of the overlaying icy shell, and any lateral variations. The TandEM mission will provide such constraints by monitoring the tidally induced fluctuation of the gravity field and topography from an orbiter and from the ground.

Seismicity on both Titan and Enceladus may be associated with tidal fluctuations, as on the Moon. Various sources such as tidally induced fracturing events, cryovolcano-tectonic activity, global contraction, trapped surface waves, natural impacts etc. may generate seismic events on Titan and Enceladus. The detection of seismic activity and localization of seismic sources would require the emplacement of seismometers by surface probes (penetrators and/or landers) that could acquire data over a sufficiently long period of time (at least a few tidal cycles: one tidal cycle $\sim 16$ days for Titan, 1.37 days for Enceladus).

Monitoring tidally induced gravity changes and seismic activity, in addition to confirming whether the satellite is tectonically active, would provide an opportunity to sound its deep interior and help characterize any threedimensional internal heterogeneity. The detection and characterization of induced magnetic fields from Titan's and Enceladus' interiors in addition to tidally-induced topographic, gravimetric and seismic signatures would provide evidence for the presence of internal liquid water layers, and place constraints on depth and composition in terms of electrolyte content. The detection of an intrinsic magnetic field on Titan (which must be quite weak based on upper-limits imposed by Cassini data [6]) would also imply operation of a selfsustained magnetic dynamo, providing important constraints on the nature of an iron-rich core.

Heat sources, cryovolcanism and eruptive processes Even though there are indications that internal outgassing has occurred on Titan and is currently occurring on Enceladus, the sources of energy and the eruptive mechanisms still remain poorly constrained [14, 29, 33, 43, 48]. Possible venting mechanisms are sudden decompression of near-surface reservoirs of liquid water [33], clathrate decomposition [20], or other cryovolcanic processes [12].

Measuring the near-surface thermal gradients and mapping the surface thermal emission on both bodies will provide key constraints on the heat flow through the icy crust. These data, combined with estimates of the ice shell thickness and tidal fluctuations, and supplemented with high resolution imaging of the icy particle jets and plume-will help determine the origin and locales of energy dissipation on Enceladus and the heat transfer mechanism [33, 43]. Temporal monitoring of the icy particle jets over time through repeated high resolution imaging will ascertain if the jetting activity is associated with tidal flexing [17, 44].

Knowledge of the composition, particularly the amount of highly volatile ices, such as ammonia, will help in understanding Enceladus' thermal history and how and when differentiation took place (http://saturn.jpl.nasa. gov/multimedia/images/image-details.cfm?imageID=2148). On both Titan and 
Enceladus, sampling of surface materials and eruptive gases will provide information on the composition of cryovolcanic materials. These data as well as remote sensing visual, infrared and radar data, will be used to assess the depth and composition of the cryomagmatic reservoir. In the case of Titan, ammonia, methane and carbon dioxide mixing ratios will help distinguish between different hypotheses about replenishing volatiles in its atmosphere $[5,12,47]$. Subsurface sounding and gravity measurements will also be used to identify cryomagmatic chambers below cryovolcanic edifices.

Early evolution: crust and atmosphere formation On Titan, the formation of the crust and of the atmosphere is intimately linked to the differentiation of the interior, which led to the formation of a discrete rock-metal core, and the crystallization of the post-accretional ammonia-water ocean [47]. A signature of the internal differentiation can be searched for in the isotopic ratios of noble gases $(\mathrm{Ar}, \mathrm{Kr}, \mathrm{Xe}, \mathrm{Ne})$ in the atmosphere and in surface materials (icy materials, deposited aerosols, hydrocarbon mixtures). On both Titan and Enceladus the abundance of radiogenically derived noble gas isotopes in the atmosphere and the gaseous plumes, respectively, will constrain the chemical exchange between the rock-metal core and the outer $\mathrm{H}_{2} \mathrm{O}$ layers. This will constrain the likelihood of silicate volcanism linked to the core and water-rock interactions during early epochs. Stable isotope ratios of actively venting volatiles bearing carbon, nitrogen and oxygen are also important in constraining the nature of volatile replenishment and atmospheric evolution.

Induction studies from combined in situ and orbital magnetometric measurements will provide information on the electric conductivity of the ocean combined with a range of depths. This will constrain the concentration of electrolytes in a current internal ocean and hence will provide some information on the early history of leaching through water-rock interactions [10, 12]. Subsurface sounding (e.g. via ground penetrating radar, seismic monitoring) in the oldest terrains (identified by impact crater statistics) may also reveal crustal layering remnant from early crust formation and subsequent recycling processes. Compositions of materials excavated by impacts will also constrain the formation process of the crust.

Measurements that are required to better constrain the deep interiors of Titan and Enceladus are delineated in Section 5.3.2.

\subsection{Astrobiological potential of Titan and Enceladus}

The Saturnian System is a key target for astrobiology. The key components of life, liquid water and organic material, are all present on moons of Saturn. Titan has an environment very rich in organics and it is often considered as one of the best targets to study prebiotic chemistry at a full planetary scale. Studies of the synthesis of organic molecules and solids on Titan may help us understand how these processes occurred on the early Earth. With the possibility of a liquid water subsurface (and the melt sheets produced by large impacts), Titan is even considered as a possible habitat for life [11, 26, 36]. 
The recent discovery on Enceladus of water ice plumes with methane and the possible presence of a large internal reservoir of liquid water containing active organic chemistry also make this small Saturnian satellite a new important planetary target for astrobiology.

\subsubsection{Similarities of Titan with the early earth}

Retracing the processes that allowed the emergence of life on Earth around four billion years ago is a difficult challenge. Our planet has drastically evolved since then, and most traces of the environmental conditions at that time have been erased. It is thus crucial for astrobiologists to find extraterrestrial locales with similarities to our planet. Such environments will provide a way to study some of the processes that occurred on the primitive Earth, when prebiotic chemistry was active.

Although Titan is much colder than the Earth, it does present many similarities with our planet (http://saturn.jpl.nasa.gov/multimedia/images/imagedetails.cfm?imageID=1105). Titan's atmosphere is made of the same main constituent, dinitrogen. It also has a similar structure from the troposphere to the ionosphere, and a surface pressure of 1.5 bar-the only case of an extraterrestrial planetary atmospheric pressure close to that of Earth. As noted in the introduction, many analogies can also be made between the role of methane on Titan and that of water on the Earth. Methane on Titan seems to play the role of water on the Earth, with a complex cycle that has yet to be fully understood.

Analogies can also be made between the current organic chemistry on Titan and the prebiotic chemistry that was active on the primitive Earth. In spite of the absence of permanent bodies of liquid water on Titan's surface, the chemistry is similar. Moreover, Titan is the only planetary body, other than the Earth with long-standing bodies of liquid on its surface (although direct observational evidence of the longevity of Titan's surface liquids remains to be obtained). Several of the organic processes which are occurring today on Titan form organic compounds which are considered as key molecules in terrestrial prebiotic chemistry, such as hydrogen cyanide $(\mathrm{HCN})$, cyanoacetylene $\left(\mathrm{HC}_{3} \mathrm{~N}\right)$ and cyanogen $\left(\mathrm{C}_{2} \mathrm{~N}_{2}\right)$. In fact, with several percent of methane in dinitrogen, the atmosphere of Titan is one of the most favourable atmospheres for prebiotic synthesis. Until recently, such an atmospheric composition was thought to be different from that of the primitive Earth. However, new modelling of the hydrogen escape in the Earth's primitive atmosphere indicates that it may have been much richer in hydrogen and methane than previously thought. This result suggests that Titan may be even more similar to the primitive Earth than expected [45].

The degree of complexity that can be reached from organic chemistry in the absence of permanent liquid water bodies on Titan's surface is still unknown, but it could be quite high. 


\subsubsection{A complex prebiotic-like chemistry}

In the atmosphere of Titan, $\mathrm{CH}_{4}$ chemistry is coupled with $\mathrm{N}_{2}$ chemistry. This combination should produce many organics in the gas and particulate phase: in particular, hydrocarbons, nitriles and complex refractory organics, The latter seem to be well modeled by the solid products-usually named "tholins"-formed in laboratory experiments ([28]; and refs included therein). We also need to consider the addition of water and oxygen ions into Titan's atmosphere. These come from a magnetospheric source that can be traced to Enceladus' plumes, and they participate in the chemical processes, forming in particular $\mathrm{CO}$ and $\mathrm{CO}_{2}$. Could these water-oxygen compounds then be locked up into aerosols?

Several organic compounds have already been detected in Titan's stratosphere. The list includes hydrocarbons (both with saturated and unsaturated chains) and nitrogen-containing organic compounds, exclusively nitriles, as expected from laboratory experiments. Since Cassini's arrival in the Saturnian system, the presence of water vapour and benzene has been unambiguously confirmed by the CIRS instrument (first detected by ISO). Surprisingly, GCMS on board Huygens did not detect a large variety of organic compounds in the low atmosphere. The mass spectra collected during the descent show that the medium-altitude and low stratosphere and the troposphere are poor in volatile organic species, with the exception of methane [27]. Condensation of such species on the aerosol particles is a probable explanation for these atmospheric characteristics. These particles, for which no direct data regarding chemical composition were previously available, were analyzed by the Huygens ACP instrument. ACP results show that the aerosol particles are made of refractory organics which release $\mathrm{HCN}$ and $\mathrm{NH}_{3}$ during pyrolysis. This supports the tholin hypothesis. From these new in situ measurements it seems very likely that the aerosol particles are made of a refractory organic nucleus, covered with condensed volatile compounds [18].

However, the nature and abundances of the condensates have not been measured. Even more importantly for astrobiology, neither the elemental composition nor the molecular structure of the refractory part of the aerosols has been determined. The potential chirality of its complex organic part is unknown.

The direct analysis of the ionosphere by the INMS instrument during the closest Cassini flybys of Titan shows the presence of many organic species, in spite of the very high altitude $(1,100-1,300 \mathrm{~km})$ [51]. Extrapolation of the INMS measurements (limited to mass up to $100 \mathrm{Da}$ ) and of CAPS data, strongly suggests that high-molecular-weight species (up to several $1,000 \mathrm{Da}$ ) may be present in the ionosphere. These new data-if confirmedrevolutionize the understanding of the organic processes occurring in Titan's atmosphere, with a strong implication that ionospheric chemistry plays a role in the formation of complex organic compounds in Titan's environment, which was predicted [3] but whose extent was not envisioned before [2, 51]. 
It is thus essential to determine the ion and neutral composition of the ionosphere with a mass range and resolution allowing the detection and characterisation of a very wide range of compounds.

In the current picture of Titan's organic chemistry, the chemical evolution of the main atmospheric constituents-dinitrogen and methane-produces mainly ethane, which accumulates on the surface or the near subsurface, and is eventually dissolved to form methane-ethane lakes and seas, and complex refractory organics which accumulate on the surface, together with condensed volatile organic compounds such as $\mathrm{HCN}$ and benzene. In spite of the low temperature, Titan is not a congealed Earth: the chemical system is not frozen. Titan is an evolving planetary body and so is its chemistry. Once deposited on Titan's surface, the aerosols and their complex organic content may follow a chemical evolution of astrobiological interest. Laboratory experiments show that, once in contact with liquid water, Titan tholins can release many compounds of biological interest, such as amino acids [19]. Such processes could be particularly favourable in regions on Titan's surface where cryovolcanism is occurring. Thus one can envision the possible presence of such compounds on Titan's surface or near subsurface. Long-term chemical evolution is impossible to study in the laboratory: in situ measurement of Titan's surface thus offers a unique opportunity to study some of the many processes which could have been involved in prebiotic chemistry, including isotopic and enantiomeric fractionation [28].

It is crucial to be able to perform a detailed chemical analysis (at the elemental, molecular, isotopic and chiral levels) of the various types of surface zones, particularly those where cryovolcanism and impact ejecta (or melt sheets) are present.

\subsubsection{Potential habitats}

All ingredients that are supposed to be necessary for life to appear and develop-liquid water, organic matter and energy-seem to be present on Titan. Indeed, interior structure models suggest that Titan, as well as Europa, Ganymede and Callisto, have maintained internal liquid water reservoirs (probably mixed with some ammonia and more speculatively sulfur). At the beginning of Titan's history, this hypothetical subsurface ocean may have been in direct contact with the atmosphere and with the internal bedrock, offering interesting analogies with the primitive Earth, and the potential implication of hydrothermal vents in terrestrial prebiotic chemistry. Consequently, it cannot be excluded that life may have emerged on or in Titan. In spite of the extreme conditions in this environment, life may have been able to adapt and to persist. Even the possible current conditions ( $\mathrm{pH}$, temperature, pressure, salt concentrations) are not incompatible with life as we know it on Earth [11]. However, the detection of a potential biological activity in the putative liquid mantle would be very challenging.

Nevertheless it seems astrobiologically essential to confirm the presence of such an internal ocean. 


\subsubsection{Enceladus}

The jets emanating from the South Pole of Enceladus are probably the most accessible samples from a liquid water environment in the outer Solar System. In addition to water ice the jets include methane, propane, acetylene, and $\mathrm{N}_{2}$ or $\mathrm{CO}$. The likely source of Enceladus' jets is a pressurized subsurface liquid reservoir (http://saturn.jpl.nasa.gov/multimedia/images/ image-details.cfm?imageID $=2026$ ). If $\mathrm{N}_{2}$ is present it may reflect thermal decomposition of ammonia associated with the subsurface liquid reservoir and may imply that the water is in contact with hot rocks-providing a source of heat as well as mineral surfaces for catalyzing reactions. If this scenario proves correct, then all the ingredients are present on Enceladus for the origin of life by chemoautotrophic pathways - a generally held model for the origin of life on Earth in deep sea vents. In this model, life on Earth began in deep sea hot springs where chemical energy was available from a mix of $\mathrm{H}_{2}, \mathrm{~S}$ and $\mathrm{Fe}$ compounds. The fact that the branches of the tree of life that are closest to the common ancestor are thermophilic has been used to argue that this reflects a thermophilic origin of life-although other explanations are possible. In the material emanating from Enceladus we can search for the specific molecules associated with such systems, including $\mathrm{H}_{2} \mathrm{H}_{2} \mathrm{~S}, \mathrm{FeS}$, etc. and the expected organic byproducts of the ecosystems that would inhabit such systems-biomarkers. Interestingly, $\mathrm{CH}_{4}$ is a key expected biological byproduct, although there are many non-biological ways $\mathrm{CH}_{4}$ can be produced. The low molecular weight organics detected by Cassini may be just one part of a suite of organics present in the plume and on the surface. Studies of the nature of these organics can tell us whether or not they are biogenic.

The molecular species likely to be produced by such a prebiotic or biotic chemistry - such as amino-acids, lipidic compounds and sugars-could be detected in the plumes of Enceladus using in situ techniques. It is also crucial to confirm the presence of liquid water reservoirs, both by remote sensing measurements and measurements made on the surface. Most of the important astrobiological questions to be covered will not be answered by CassiniHuygens and require a dedicated new mission to Titan and Enceladus.

\section{Mission profile/scenario}

\subsection{Mission concept}

The baseline mission concept is for two moderately-sized spacecraft to provide both in situ (near or at the surface) orbital and surface science measurements at both Titan and Enceladus, via establishment of a novel, but practical, orbit, which cycles around these two moons (Fig. 2). This mission architecture could provide a cost-effective solution, which is efficient in launch mass, very flexible, and provides a significant element of fault tolerance. In addition, this 
Fig. 2 TandEM flight elements with orbital information

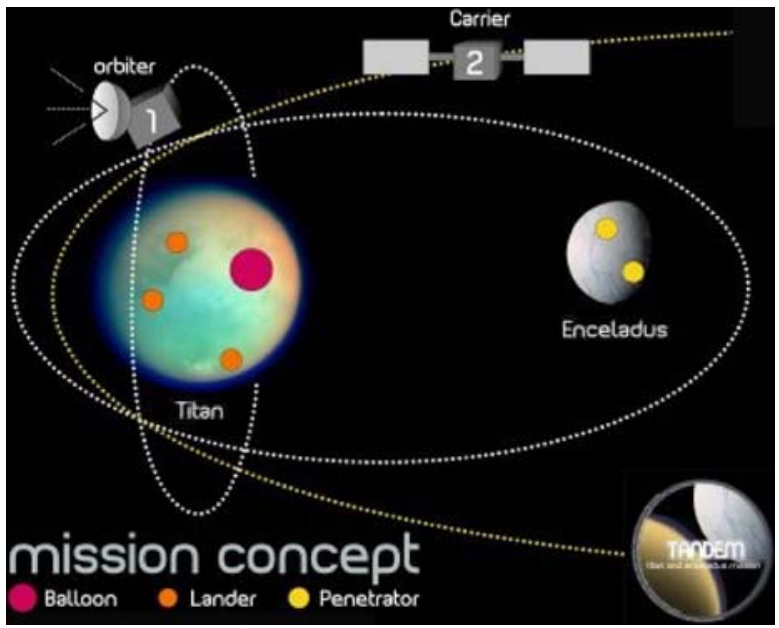

architecture-through its multi-element nature-lends itself to international collaboration.

\subsubsection{Baseline architecture}

While TandEM could be implemented through a number of propulsion system options, in our proposal, we concentrated on technologies which provide low risk to the mission. The baseline architecture uses chemical propulsion, while future studies could investigate other options (SEP, aerocapture, aerobraking, etc.).

Our baseline mission concept then considers two spacecraft to be launched around 2018 or later on one or two launch vehicles (to be assessed):

1. the Titan-Enceladus orbiter (carrying the Enceladus penetrators), and

2. the carrier for the Titan in situ investigation elements (the Titan Montgolfière/balloon and the three lander/mini-probes)

A possible TandEM mission timeline chart is given hereafter in Table 2.

\subsubsection{Key mission phases}

The orbiter will perform several flybys of the two moons (Titan and Enceladus) early in the mission and will deliver the Enceladus landers/penetrators. The carrier spacecraft would deliver the Titan in situ assets, including the Titan hot air balloon with a mass allocation of $\sim 265 \pm 30 \mathrm{~kg}$, and up to three entry probes with a mass allocation of $\sim 500 \mathrm{~kg}$. Future studies could consider converting this carrier spacecraft into an orbiter, thus allowing for telecom redundancy 
Table 2 TandEM mission possible timeline

\begin{tabular}{|c|c|c|}
\hline \multicolumn{3}{|l|}{ Possible timeline } \\
\hline Proposal pre-selected & Oct. 2007 & \\
\hline Internal assessment phase in parallel with Laplace & Nov. 2007-Oct. 2008 & \\
\hline Industrial assessment phase and TDP definition & Aug. 2008-June 2009 & \\
\hline SPC down selection to two missions & Nov. 2009 & \\
\hline Competitive definition phase & Jan. 2010-June 2011 & \\
\hline Technology development & 2009-2014 & \\
\hline Consolidation of international collaboration & 2009-2014 & \\
\hline SPC selection of first mission & Nov. 2011 & \\
\hline Start of industrial phase & 2011/2013 & \\
\hline Development (7 years) & $2011 / 2013-2018 / 2020$ & \\
\hline Launch & $\mathrm{L}$ & 2018 or later \\
\hline Interplanetary trajectory & $\mathrm{L}$ to $\mathrm{L}+7-9$ years & 2025-2027 or later \\
\hline Arrival at Saturn & A & 2027 or later \\
\hline Phasing for Enceladus mission phase & $\mathrm{A}-\mathrm{A}+1$ year & $2027-2028$ or later \\
\hline Prime Titan mission phase & Duration $\sim 2$ years $(\mathrm{TBD})$ & 2028-2030 or later \\
\hline Extended mission phase at Titan & 2 years & 2030-2032 or later \\
\hline
\end{tabular}

from the surface assets and some interesting science measurements (e.g., occultations using the telecom link between the two orbiting spacecraft). The orbiter would provide telecom relay between the Titan in situ elements and Earth.

Launch and cruise phase for both spacecraft In our baseline architecture, both spacecraft would be sent to Saturn on a multiple inner planets gravity assist, using chemical propulsion for the cruise phase, employing swing-bys of inner Solar System planets, and the possibility for cruise phase science. An ExoMarslike launch but with Atlas V-521 could deliver $\sim 1,700 \mathrm{~kg}$ mass to Saturn. The orbiter, initially acting as the Titan-Enceladus cycler, then as a Titan science and telecom orbiter, would deliver up to two Enceladus penetrators, with an assumed mass allocation of $\sim 100 \mathrm{~kg}$ each. As for the second spacecraft, the most effective scenario is that planned for the ExoMars mission, which would allow a $1,660 \mathrm{~kg}$ mass at Saturn with $\sim 800 \mathrm{~kg}$ still available for the entry probes. This large mass margin would allow for spacecraft growth and/or payload increase, or alternatively could result in a smaller launch vehicle-an option, which is based on mission cost and programmatics.

Insertion into Titan-Enceladus cycling orbit establishment The baseline architecture assumes chemical propulsion for both the cruise phase and for following Saturn orbit insertion (SOI). It is then followed by four Titan swingbys to bring the period down to that of the cyclical phase (3:7 resonance between Titan and Enceladus). For SOI, the option for aerocapture at Titan will be studied as an alternative to chemical propulsion, for example with respect to fuel mass savings $(\sim 1.8 \mathrm{~km} / \mathrm{s})$, technology impact, reduced time to be captured into a 2:1 Titan-Enceladus 32-days resonant orbit in a single pass 
in comparison to $\sim 130$ days with chemical propulsion (Fig. 3). Aerobraking is another flight-qualified technique which could be envisaged to save mass.

Titan-Enceladus cycling orbit operations The Titan-Enceladus Orbiter would deliver up to two Enceladus landers/penetrators, which would perform in situ seismic, temperature, and possibly compositional measurements. The collected data would be relayed back to Earth through the T-E orbiter during its Enceladus flybys. Around the beginning of this phase the orbiter releases (simultaneously or in separate flybys) the Enceladus mini-probes/penetrators to provide the Enceladus surface investigations via data which are relayed to the Orbiter during its Enceladus flybys. Regular flybys of Enceladus would occur every $\sim 8.25$ days, allowing for considerable targeting flexibility (e.g. some or all could encounter the South Pole plume). The baseline scenario assumes 12 Enceladus and correspondingly two Titan swing-bys, followed by three to four swing-bys of Titan on the way to Titan orbit insertion (Fig. 4). This phase provides coordinated Enceladus surface and flyby science; flyby Titan science; other Saturnian science, and the serendipitous ability to directly investigate the possible transport of (organic) material from Enceladus to Titan and indirectly to other parts of the Saturnian system.

Titan orbit Coordinated Titan orbital science, Montgolfière operations and surface probe investigations (for $\sim 1-2$ years) will take place after the orbital insertion around Titan. Indeed, at the end of the previous phase, the Orbiter is manoeuvred to a polar orbit solely around Titan to perform optimised Titan orbital science and provide telecom support to the Titan in situ elements. Specifically, the orbiter will provide high data rate relay communications from the Titan balloon and the small probes to the Earth. The mission is designed so that the Titan Orbiter is established in Titan polar orbit before the flyby carrier delivers the Titan probes. This would leave time for the Orbiter observations of Titan and orbit optimization, in preparation for the in situ measurement support.
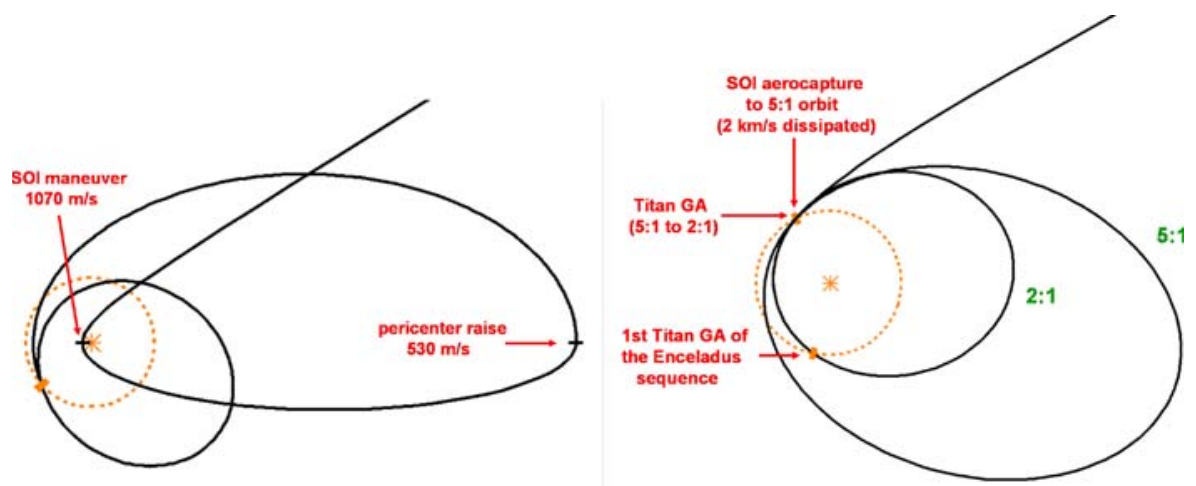

Fig. 3 TandEM orbit insertion: chemical (left) and using aerocapture (right) 


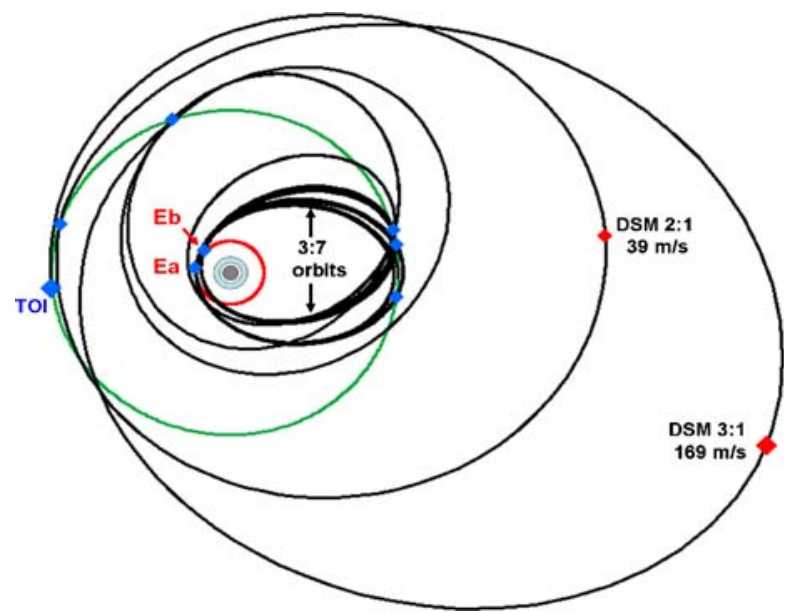

Fig. 4 Enceladus-Titan phase of the TandEM mission. Following insertion, a series of three Titan gravity assist injects the spacecraft into an orbit with a period of 6.85 days, which is five times the period of Enceladus (1.37 days). After seven encounters with Enceladus ( $E a)$, a small adjustment makes it possible to encounter Titan again, as the elapsed time (47.95 days) is very close to three Titan periods (47.84 days). A second series of encounters $(E b)$ can be implemented, extending the Enceladus phase to 96 days with up to 14 fly-bys. A series of five Titan fly-bys and two small deep space manoeuvres implementing a DVGA strategy reduce the relative velocity to $965 \mathrm{~m} / \mathrm{s}$, at which stage a $420 \mathrm{~m} / \mathrm{s}$ insertion manoeuvre results in a 1 day elliptical orbit around Titan. The period can be further reduced by aerobraking

\subsection{Earth-based science segment of the mission}

Earth-based and Earth-bound observations will be conducted on two distinctive sets of targets relevant to TandEM: the mission objects (Titan, Enceladus and their environment) and the TandEM mission spacecraft. The former will help to prepare the mission science operations and then interpret the data from in situ observations and experiments. These "traditional" astronomical observations will be conducted in a broad range of wavelengths, from highenergy domain to low radio frequencies using a variety of Earth-based and Earth-bound facilities.

The latter will include VLBI and Doppler tracking of the spacecraft aimed at achieving very high accuracy of trajectory characterization of all mission spacecraft in various phases of their operations and localisation of in situ measurements required for the mission objectives. In addition, the radio telescopes involved in VLBI tracking will provide a backup "eavesdropping" support to the nominal mission communication scenario enabling receipt of low data-rate signals during critical and/or high scientific value events of the mission.

\subsubsection{Astronomy support observations of the TandEM mission objects}

The TandEM Earth-based observing campaign will follow the example of Cassini-Huygens mission. The data obtained by these observations will 
provide synergic to in situ TandEM measurements. While the specific scientific programme of astronomical observations in support to the TandEM mission is to reflect the mission programme, one should anticipate particular attention to the following topics:

- Long-term seasonal monitoring of all objects of the Saturnian system;

- Characterisation of Titan atmosphere using continuum (visual, IR) and spectral line (UV, visual, IR, millimetre and sub-millimetre domains) of the Titan atmosphere;

- Visual and IR characterisation of the surface of Enceladus;

- Multi-band monitoring of non-thermal radiation of the iono- and magnetospheres of Saturn and other constituencies of the Saturnian system.

The astronomical observations will be conducted in many cases using national and international facilities, accessible via open proposal peer-review mechanism. The TandEM mission will coordinate Earth-based astronomical observing campaign within its remit and in compliance with the access mechanisms of the observing facilities.

\subsubsection{VLBI and Doppler tracking segment of the TANDEM mission}

The Earth-based global network of radio telescopes and processing facilities (correlators) will conduct Planetary Radio Interferometry and Doppler Experiment (PRIDE) aimed at providing ultra-precise estimate of the state-vector of the TandEM mission spacecraft. It will be based on the heritage of the Huygens Doppler Wind Experiment (DWE) and Very Large Baseline Interferometry (VLBI) tracking experiments. Today's technology and very conservative projection of capabilities of VLBI radio telescopes for the next two decades lead to the following guaranteed $1 \sigma$ accuracy of positional measurements: $500 \mathrm{~m}$ based on S-band (2 GHz signal), $100 \mathrm{~m}$ at X-band $(8 \mathrm{GHz})$ and $30 \mathrm{~m}$ at Ka-band (32 GHz). VLBI tracking of all mission space-based elements poses minimal requirements on the on-board instrumentation, namely (1) optimisation of the transmitters signal spectra and (2) availability of a reasonably stable on-board local oscillator within the on-board radio transmitting sub-system.

\subsubsection{Direct-to-earth data transmission}

The nominal mission scenario assumes transmission of the science and housekeeping data from the Titan and Enceladus probes via relay by the orbiter (carrier spacecraft). Indeed, the amount of data produced by the probes, or the Titan balloon (e.g. images) will require a high-capacity radio relay system. However, as an efficient backup able to provide support to critical mission operations and experiments, a low data-rate link can be achieved with the nominal transmission from the probe(s) and received by the large Earth-based radio telescopes. The most attractive option of DtE would involve the Square Kilometre Array (SKA) as the Earth-based facility able to operate at the $\mathrm{S}$ band $(2.3 \mathrm{GHz})$. The facility is expected to be fully operational in 2020 . 
As shown by preliminary assessment estimates, SKA will be able to receive data streams from the TandEM mission spacecraft at the rate of 30-100 bps. Implementation of the TandEM DtE using SKA does not require any specific modifications of the on-board radio systems, but does require optimisation of the mission scenario in order to achieve direct visibility "S/C-SKA" during critical mission events. Detailed trade-offs of the TandEM DtE regime will be investigated during the project assessment study.

\section{Proposed payload instrument complement}

This study will investigate strategies to combine instrument concepts to minimize resource requirements and achieve multiple measurement requirements. Miniaturization strategies that maintain functionality will be investigated. Heritage will of course be important, but new technology and innovative instrument concepts should be encouraged. A list of a baseline strawman payload is given in this section for each component. Note however, that this is a preliminary list that will be refined and justified during the next study phase, if the mission is selected. We will also look into instrument strategies that will fulfil multiple science goals of both Titan and Enceladus science. More work is required from the science community to arrive at well-defined strawman payload for each element of the mission.

\subsection{Titan as a system}

For the upper atmosphere and magnetospheric interactions scientific objectives, the required measurements are described in Table 3. Some of the required orbiter in situ measurements need coverage of $4 \pi$ viewing angle (pitch-angle) with high time resolution $(6 \mathrm{rpm})$. It is therefore necessary to put the orbiter in a spinning science phase for at least two Titan days (32 days) together with an additional commissioning period just before this spin-phase. This spin-phase should be part of the Titan polar and elliptical orbit phase when the periapsis is lowered to $800 \mathrm{~km}$ (or as low as possible).

For Titan's neutral atmosphere the objectives that we delineate in Section 3.1.2 point to the measurements and the instrumentation proposed in Table 4.

The objectives of Titan Surface Sciences (Section 3.1.3) are not being addressed by Cassini. Indeed, with the exception of the small region around the Huygens landing site, Cassini's optical and radar resolution is inadequate to characterize small scale and possibly dynamic geological features (methane geysers, cryo-flows, stream deposits, dunes etc.) See Fig. 5.

Hence the measurements required to investigate the above objectives include:

- high-resolution global optical infrared stereo mapping and radar surface detection (resolution $<100 \mathrm{~m}$ ) 
Table 3 Instrumentation required for the Titan upper atmosphere and magnetospheric measurements

\begin{tabular}{ll}
\hline Key measurements & Instruments \\
\hline Densities of major neutral species $\left(\mathrm{N}_{2}, \mathrm{CH}_{4}, \mathrm{H}_{2}\right)$ & UV spectrometer \\
& Ion and neutral mass spectrometer (INMS) \\
& Accelerometer \\
& Radio science \\
& Auroral and airglow photometer \\
Densities of aerosols, positive and negative ions & INMS \\
$(10-10,000$ amu $)$, stable and reactive $\mathrm{H}, \mathrm{C}, \mathrm{N}$ & Aerosol analyser \\
containing neutral species with resolution & Millimetre and sub-millimetre spectrometer
\end{tabular}
of $0.01 \mathrm{amu}$

Direct measurements of neutral winds ion velocities

Vertical profiles of electron and ion temperatures

Langmuir probe

Electric fields and plasma waves

Ion drift meter

Millimetre and sub-millimetre spectrometer

Vertical profiles of neutral temperature

Langmuir probe

Radio science

(above $400 \mathrm{~km}$ )

Millimetre and sub-millimetre spectrometer

UV spectrometer

INMS

Accelerometer

Exospheric structure including transition region,

Radio science

corona, spatial and temporal variability, escape kinetics and rates of important atmospheric species, ENA formation

Ionisation and heating processes of Titan's atmosphere/ionosphere (HCN)

Energetic neutral atom imager and composition analyser (ENA)

UV spectrometer

INMS

Plasma package ${ }^{\mathrm{a}}$

INMS

Auroral and airglow photometer

Radio science

ENA

Photochemistry during solar eclipse of Titan by Saturn

UV spectrometer

INMS

Plasma package ${ }^{\mathrm{a}}$

Radio science

Measurements of global plasma and magnetic field structure of induced magnetosphere for different conditions of Saturn Local Time and magnetospheric dynamics out to $\sim 10 \mathrm{R}_{T}$

Measurements concerning ion loss rates for different chemical species through the tail/ wake region under varying magnetospheric condition

Unexplored low-altitude structure of the magnetic ionopause and below

Study particle populations near magnetic ionopause

Plasma package

ENA

Plasma package ${ }^{\mathrm{a}}$

INMS

Radio science

Dual magnetometer

Plasma analyser

Langmuir probe

Atmosphere measurements must cover the globe and differing solar illumination.

aThe Plasma package can be a highly integrated payload suite (HIP) that consists of a plasma analyser (electrons and ions), dual magnetometer, energetic ion and electron detector, dual Langmuir probe, electric field and plasma waves, ion drift meter and a search coil magnetometer. 
Table 4 Instrumentation required for the Titan neutral atmosphere measurements

\begin{tabular}{ll}
\hline Key measurements & Instruments \\
\hline Temperature, pressure and methane & GCMS \\
and ethane humidity at different & ASI/MET \\
latitudes and seasons, with high & Radio science \\
fidelity near the surface & \\
Wind from the surface to the thermosphere & Microwave sounder $(100-700 \mathrm{~km}$, error $10 \mathrm{~m} / \mathrm{s})$ \\
& IR spectrometer $(70-500 \mathrm{~km})$ \\
& Accelerometer $($ lower troposphere, error $<1 \mathrm{~m} / \mathrm{s})$ \\
& ASI/MET (surface, error $<1 \mathrm{~m} / \mathrm{s})$ \\
Structure of clouds and precipitation & Visible and NIR camera and mapping spectrometer \\
& Nephelometer \\
& Optical rain gauge \\
& Electric field sensor \\
Opacity structure and scattering & Nephelometer \\
characteristics of haze & Visible and NIR camera and mapping spectrometer \\
Evaporation rates, temperature & GCMS \\
and winds over lakes & ASI/MET \\
Composition of the surface and its & Heat flow/physical properties probe \\
thermal properties & Visible and NIR camera and mapping spectrometer \\
& Tunable laser spectrometer \\
& Accelerometer \\
& Subsurface camera/microscope (mineralogy) \\
Composition of the haze and its & Subsurface spectrometer \\
building molecules & Aerosol analyser \\
Solar partitioning of sunlight and thermal & Electric field sensor \\
cooling at different latitudes and seasons & Visible and NIR camera and mapping spectrometer \\
Latitudinal and vertical distribution of minor IR spectrometer \\
hydrocarbons, nitriles and oxygen-bearing & Microwave sounder \\
species and its temporal variation & GCMS \\
& Visible and NIR camera and mapping spectrometer \\
\hline &
\end{tabular}

- highest-resolution $(<1 \mathrm{~m})$ infrared imaging from a near-surface platform (required in any case to select appropriate sampling sites for surface chemistry)

Furthermore, no detailed surface composition mapping was possible with Cassini-Huygens, nor are there measurements of the depth of deposits available to permit a good organic inventory to be determined. Therefore, the following measurement scenario is required:

- global mapping from an orbiter

- regional surface investigations from a near surface platform-balloon

- in situ measurements from a set of local landed stations/micro-penetrators or using tethers to transport to the balloon subject to further study

with the instrumentation given in Table 5. 


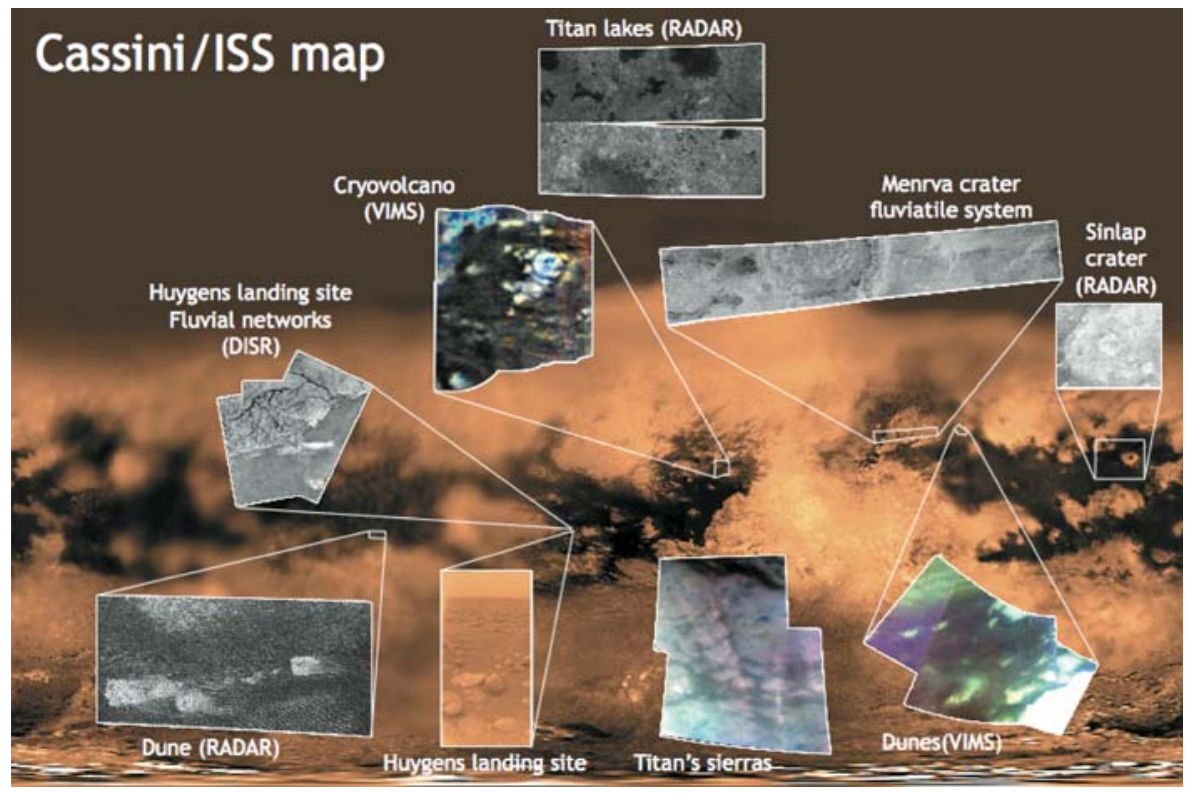

Fig. 5 The diversity of Titan's surface: geological features discovered by Cassini/Huygens which remain to be explained. Future landing locations will be selected among such sites (Credit: Univ. Nantes and CIGAL/LESIA)

\subsection{Enceladus}

Our prime science objectives to achieve with regards to Enceladus are to:

- understand the origin, nature and properties of the plume

- reveal the existence, depth and extent of sub-surface liquid water

- search for signs of past/present life and in the process of resolving these issues to also:

- characterize the surface and the interior

- characterize the impact of Enceladus on the magnetosphere, other satellites and the ring structure

In order to achieve these science objectives the following measurements via the orbiter are required (Table 6).

\subsection{Origin, interior and evolution}

\subsubsection{Origin and evolution}

Several observational tests may help address the question of Titan and Enceladus' origin and evolution. They are described hereafter in Table 7. 
Table 5 Instrumentation required for the Titan surface measurements

\begin{tabular}{ll}
\hline Key measurements & Instruments \\
\hline $\begin{array}{c}\text { High-resolution global optical infrared stereo } \\
\text { mapping and radar surface detection } \\
\text { (resolution }<100 \mathrm{~m})\end{array}$ & $\begin{array}{l}\text { Infrared multispectral stereo camera } \\
\text { Radar imaging }\end{array}$ \\
$\begin{array}{c}\text { Highest-resolution }(<1 \mathrm{~m}) \text { infrared imaging } \\
\text { from balloon }\end{array}$ & NIR-microscope/close-up imager \\
$\begin{array}{c}\text { Composition of the surface, structure, thermal, } \\
\text { and electrical properties }\end{array}$ & Heat flow/physical properties probe \\
& Accelerometer \\
& Subsurface camera/microscope (mineralogy) \\
& Spectrometer \\
High-mass-resolution in situ measurements & Descent camera \\
of the surface material (molecular and isotopic) & X-ray spectrometer \\
from a variety of locations & Raman-LIBS spectrometer \\
Compositional context mapping from balloon & Subsurface spectrometer \\
Global compositional mapping (resolution $<1 \mathrm{~km})$ & NIR camera and mapping spectrometer \\
Depth of surface deposits & Radar sounder \\
& Accelerometer \\
& Seismometer \\
$\begin{array}{l}\text { Global wind pattern and its seasonal variation } \\
\text { Physical properties of the interior (internal }\end{array}$ & ASI/MET \\
structure, intrinsic heat flow, thermal and & Heat flow/physical properties probe \\
chemical boundary layers) & Seismometer \\
Surface energy exchange (radiation, temperature, & Radiation dosimeter \\
chemical boundary & ASI/MET \\
& GCMS \\
& Optical rain gauge \\
Astrobiology detections (habitable subsurface & Heat flow/physical properties probe \\
biotic and pre-biotic water; chemistry, & Seismometer \\
life forms) & Subsurface camera/microscope \\
& Spectrometer \\
\hline &
\end{tabular}

\subsubsection{Interiors}

The required measurements for addressing the internal structure goals (as presented in Section 3.3.2) are given in Table 8.

This series of measurements will permit to retrieve (a) the depth and composition of the putative interior liquid layer, (b) the size and state of the rock-metal core, (c) the structure of the crust and the depth of liquid reservoir, (d) the internal differentiation process as well as the formation and recycling process of the atmosphere and the crust.

\subsection{Astrobiology measurements}

Instrument suggestions for astrobiological studies (to be implemented for both satellites) are given in Table 9. Other techniques, similar to those under development for Mars organics analysis (such as the Urey experiment) can be envisaged. 
Table 6 Instrumentation required for Enceladus orbiting, and Enceladus and Titan surface and interior measurements

\begin{tabular}{ll}
\hline Key measurements & Instruments \\
\hline $\begin{array}{l}\text { Map the physical and chemical surface } \\
\text { properties and relate them to surface } \\
\text { processes, organics and volatiles and } \\
\text { physical properties of water ice; passive } \\
\text { multispectral remote sensing observations } \\
\text { of the surface and plumes; infrared remote }\end{array}$ & \\
sensing temperature maps of cracks and & \\
cervices with vents & \\
Remote imagery over time of the plume at & \\
a variety of phase angles and distances; stereo & \\
imaging to measure shape of Enceladus; long & \\
term imaging of Enceladus from a stable orbit & \\
around Titan with 50-250 m resolution & \\
In situ particle, dust, magnetic field observations & \\
with composition capability of high sensitivity & Magnetometer camera $(10 \mathrm{~m} / \mathrm{pixel})$ \\
and mass resolution & Energetic particle detector \\
& Energetic neutral atom imager \\
& Plasmation neutral mass spectrometer \\
& Dust detector \\
& Gas analyser \\
\hline
\end{tabular}

Table 7 Instrumentation required for the Titan and Enceladus origin measurements

\begin{tabular}{|c|c|}
\hline Key measurements & Instruments \\
\hline $\begin{array}{l}\text { Isotopic ratios in major species }(\mathrm{H}, \mathrm{C}, \mathrm{N}, \mathrm{O}) \\
\text { in surface solid materials (water ice, } \\
\text { deposited aerosols, hydrocarbon mixtures) } \\
\text { and in gaseous compounds }\end{array}$ & $\begin{array}{l}\text { Gas chromatograph mass spectrometer } \\
\text { (GCMS), capable of measuring stable isotopes } \\
\text { and incl. shared pyrolysis and chemical extraction } \\
\text { Ion and neutral mass spectrometer }\end{array}$ \\
\hline $\begin{array}{l}\text { Noble gas abundances and isotopic ratio } \\
\text { (Ar, } \mathrm{Kr}, \mathrm{Xe}, \mathrm{Ne}) \text { with } 100 \text { to } 1,000 \text { times } \\
\text { higher sensibility than those measured by } \\
\text { Cassini-Huygens }\end{array}$ & GCMS (as above) \\
\hline $\begin{array}{l}\text { Sulfur compounds in surface materials } \\
\left(\mathrm{H}_{2} \mathrm{~S}, \mathrm{OCS}\right)\end{array}$ & GCMS (as above) \\
\hline
\end{tabular}

Table 8 Instrumentation required for the Titan and Enceladus interior measurements

\begin{tabular}{ll}
\hline Key measurements & Instruments \\
\hline Mapping topography, gravity and & Radar or laser altimeter \\
magnetic fields on global and local & Radio science subsystem, gravity gradiometer \\
scales & Magnetometer \\
Seismic and tidal survey & $\begin{array}{l}\text { Seismometer } \\
\text { Gravity gradiometer } \\
\text { Radar or laser altimeter }\end{array}$ \\
Subsurface sounding, crustal layering and & Ground penetrating radar \\
liquid reservoirs & Thermal sensing package \\
Near-surface thermal gradient and & \\
thermophysical properties & Surface sample analysis package \\
Composition of surface materials, of & GCMS \\
cryovolcanic magma and gases & Vis-NIR mapping spectrometer \\
(especially C, N, O isotopic ratios) & Radar imaging \\
& GCMS \\
\hline Radiogenically-derived noble gas abundances &
\end{tabular}


Table 9 Instrumentation required for the Titan and Enceladus astrobiology measurements

\begin{tabular}{|c|c|}
\hline Key measurements & Instruments \\
\hline $\begin{array}{l}\text { Ionosphere: ion and neutral composition } \\
\text { within a high mass range (several 1,000 Da) }\end{array}$ & $\begin{array}{l}\text { Orbiter } \\
1-2,000 \text { Da high resolution MS (TOF) }\end{array}$ \\
\hline $\begin{array}{l}\text { Stratosphere-troposphere: high } \\
\text { sensitivity (sub-ppb level) molecular and }\end{array}$ & Particle collector and analyser? \\
\hline $\begin{array}{l}\text { sensitivity (sub-ppb level) molecular and } \\
\text { isotopic analysis of the gas phase. Chemical }\end{array}$ & Balloon \\
\hline alysis of the gas phase. Chemical & Altimeter \\
\hline on of the aerosols: organic and & Stable isotope mass spectrometer \\
\hline $\begin{array}{l}\text { inorganic analysis, elemental, molecular and } \\
\text { isotopic analysis of the aerosols-vertical and } \\
\text { latitudinal variations }\end{array}$ & $\begin{array}{l}\text { Gas chromatograph-high resolution } \\
\text { mass spectrometer with capabilities for } \\
\text { analyzing refractory materials (laser }\end{array}$ \\
\hline $\begin{array}{l}\text { Surface: organic and inorganic analysis-elemental, } \\
\text { molecular, isotopic and chiral analysis } \\
\text { of the surface materials }\end{array}$ & $\begin{array}{l}\text { desorption, chemical derivatization, } \\
\text { differential thermal analyser-pyrolyser, } \\
\text { chemolysis) }\end{array}$ \\
\hline $\begin{array}{l}\text { Subsurface: from penetrators and/or } \\
\text { by analyzing surface materials } \\
\text { ejected from subsurface }\end{array}$ & $\begin{array}{l}\text { Subcritical water extractor-microcapillary } \\
\text { electrophoresis system } \\
\text { Probes/landers }\end{array}$ \\
\hline $\begin{array}{l}\text { Hydrocarbon related mineralogy for surface } \\
\text { and subsurface }\end{array}$ & $\begin{array}{l}\text { IR stereo camera/spectrometer for context } \\
\text { X-ray fluorescence spectrometer }\end{array}$ \\
\hline $\begin{array}{l}\text { Need to analyze different areas: bright } \\
\text { and dark regions, lake/damp playa/ } \\
\text { shoreline/dune field }\end{array}$ & $\begin{array}{l}\text { Stable isotope mass spectrometer } \\
\text { Gas chromatograph mass spectrometer with } \\
\text { capabilities for analysing refractory } \\
\text { material, and chiral GC columns } \\
\text { Subcritical water extractor-microcapillary } \\
\text { electrophoresis system } \\
\text { Raman/LIBS spectrometer } \\
\text { Drilling capability to analyse the subsurface } \\
\text { and/or melting system }\end{array}$ \\
\hline $\begin{array}{l}\text { Molecular analysis of the plumes and } \\
\text { search for a subsurface ocean }\end{array}$ & $\begin{array}{l}\text { Orbiter } \\
1-2,000 \text { Da high resolution MS (TOF) } \\
\text { Particles collector and analyser? } \\
\text { See Section } 3.2\end{array}$ \\
\hline $\begin{array}{l}\text { Search for evidences of internal water ocean and } \\
\text { information on its properties }\end{array}$ & See Sections 3.1 and 3.3 \\
\hline $\begin{array}{l}\text { Search for episodical liquid water bodies } \\
\text { on the surface }\end{array}$ & \\
\hline $\begin{array}{l}\text { Search for molecular, isotopic }(\mathrm{C}, \mathrm{S} \text { and } \mathrm{O}) \\
\text { and chiral biosignatures }\end{array}$ & $\begin{array}{l}\text { Penetrators/probes } \\
\text { Stable isotope mass spectrometer } \\
\text { GC-MS with chiral columns }\end{array}$ \\
\hline $\begin{array}{l}\text { Search for molecular, isotopic and chiral (?) } \\
\text { biosignatures }\end{array}$ & $\begin{array}{l}\text { Orbiter } \\
1-2,000 \text { Da high resolution MS (TOF) } \\
\text { Particles collector and analyser? }\end{array}$ \\
\hline
\end{tabular}

\subsection{Preliminary strawman payload}

A traceability matrix was constructed from the science goals prioritization and the associated instrumentation. The strawman instrument payload proposed hereafter provides a strong set of crosscutting complementary observational capabilities, as determined by our traceability matrix. Cameras, spectrometers, magnetometers, radar, radio science, seismometers, organic matter and surface composition analyzers, and a host of new conceptual instruments will scan all 
spectral ranges and return data of high level of detail and quality. The combination of orbiter and in situ elements provides opportunities for both large and small payloads, engaging a potentially wider community of instrument providers from a variety of member states. The heritage of previously successful missions such as Cassini-Huygens, Rosetta, Venus and Mars Express, etc. as well as new ones currently under study (such as ExoMars, etc.) will be extremely beneficial to the definition of the technological feasibility and maturity of the proposed concept.

A TandEM orbiter strawman payload could include such instruments as an advanced ion and neutral mass spectrometer, a thermal IR spectrometer, a dual magnetometer, a microgradiometer, a sub-surface radar, Radar and laser altimeters, and SAR, a radio science experiment, a UV spectrometer, a visible and near-IR camera and mapping spectrometer (in the 0.9-6 $\mu \mathrm{m}$ range). Other possibilities include a Dual Langmuir probe, a microwave (submm) sounder/spectrometer, a neutral analyser (LENA) and a plasma analyser (for electrons and ions).

For a Titan aerial platform, a GCMS (with stable isotope measuring capabilities), a visible and near-IR camera and mapping spectrometer, an atmospheric structure instrument (MET) and a sub-surface Radar (GPR) would beaccording to the traceability matrix-among the main instruments to be on board.

On the Titan mini-Huygens landing probes one would obviously want to have a gas analyzer with chemical sensors, a gas chromatograph mass spectrometer (with stable isotope) and a radio science experiment, as well as a sub-surface radar, a microscope/lose-up imager and a visible and nearIR high-resolution camera and mapping spectrometer depending on the mass allocation permitted.

For the Enceladus penetrators and in order to perform such key measurements as sub-surface chemistry, searching for water and organics, internal body and core structure, surface activity, morphology and landing site context, etc. one might envisage chemical and thermal sensors, seismometers, descent camera, accelerometers, etc.

\section{Basic spacecraft key factors and technology issues}

The TandEM mission will be built around a central core of an orbiter and a number of in situ elements, accommodating a core group of instruments. Specifically, our baseline architecture assumes the use of two smaller launch vehicles. The first one-a Titan-Enceladus orbiter-would also deliver the Enceladus penetrator(s), while a second Titan flyby/carrier spacecraft would accommodate the Titan hot air balloon and the Titan mini-probes. Alternatively, a large single launch vehicle could be used, but this introduces other complexities to the mission. Aerobraking within Titan's atmosphere will be given serious consideration to minimize resource requirements and risk. Our baseline approach will allow the orbiter to be used initially for both Enceladus 
and Titan science, with a terminating orbit around Titan. A common set of instruments will be used for both Enceladus and Titan orbiter science to reduce mission cost.

The initial orbiter phase would be optimized for Enceladus science via numerous flybys (i.e., cycler orbits) of Enceladus' southern plumes, using advanced remote sensing, and particle and field instrument packages. Additionally, such a cycler orbit will allow direct in situ study of the possible transport of (organic) material between Enceladus and Titan, and indirectly to other parts of the Saturnian system. During this phase, the Enceladus penetrator(s) would be released to perform Enceladus surface investigations via data relayed to the orbiter on its flybys. The Titan balloon and miniprobes would be phased to arrive after the orbiter achieves circular Titan orbit, and the orbiter has first completed an initial reconnaissance phase to support balloon and probe science; study Titan's upper atmosphere and winds remotely; measure the internal structure using altimetry and radio science data and finally study the Titan-magnetosphere interaction using both in situ and remote sensing techniques.

The Titan aerial probe is conceived as a Montgolfière hot air balloon concept, which could stay aloft for several years within Titan's atmosphere and perform touch-and-go sample acquisition from various surface locations. Combining the RTG-heated hot air balloon with a tether-suspended sampling mechanism-attached to the gondola-would allow for global in situ measurements of Titan's lower atmosphere and surface. Based on topographical maps of the surface, atmospheric winds and global compositional maps of the surface from the orbiter, the hot air balloon will be able to navigate safely and have its science mission operations optimized by the time it reaches the surface. The aerial probe, once in Titan's atmosphere, will navigate nominally at altitudes of $\sim 10 \mathrm{~km}$, with ceilings at 3 and $25 \mathrm{~km}$ above the surface, and provide global measurements of Titan's lower atmosphere and winds, as well as close up images of the surface and its composition.

Mini-probes (either descent and landing modules launched from the carrier or landers/penetrators deployed from the Montgolfière) will be used for a serendipitous investigation of surface features, as well as atmospheric investigations at different locations than covered by the balloon. Specific terrain targeting and co-temporality will be assured to allow for a small seismic network to be established. Micro-penetrators are also well suited to investigating terrains, which are difficult for soft landers. Further study is required to determine exact probe numbers and configurations according to available mass, capabilities and risk.

This mission will require new technologies and capabilities so that the science goals can be achieved within the cost cap, schedule, and acceptable risks. Miniaturization, while maintaining functionality of spacecraft and science package, will be part of our Technology Development Plan (TDP). International participation will play a key role in achieving all the science goals of this mission within acceptable cost to all partners. We will sub-divide 
the science payloads into remote sensing packages and in situ packages with instrument PIs for each to simplify management structure and allow for optimization of resources and cost within each of the instrument package teams. For example, we will give serious consideration to the development of an integrated remote sensing package for the orbiter with a common telescope to minimize resource and cost, and allow long-term observations of the Saturn system to be made from a stable Titan orbit. Such observations could be performed through the extended mission phase.

Therefore, in this section, we identify a baseline launch mass and sub-system structure to provide an overall mass, volume and power budget from which rough order of magnitude (ROM) costs can be identified. Approved engineering models will be used to assess resource requirements for such a scenario and additional capabilities, if possible within acceptable cost and resource margins, will be added to the payload. This will then provide a framework around which our proposed mission concept will be built. The above mission concept will allow us to identify key areas for technology development and corresponding development of a technology plan.

\subsection{Orbiter}

Here, we identify the key pieces of the Titan-Enceladus orbiter with a baseline science payload. Our baseline configuration uses chemical propulsion only for the cruise phase and for Saturn orbit insertion (SOI) with a $\Delta V$ of $\sim 2 \mathrm{~km} / \mathrm{s}$. In addition, the Titan-Enceladus cycling phase, plus Titan orbit insertion (TOI) would require an additional $\Delta V$ of $\sim 1 \mathrm{~km} / \mathrm{s}$. As a mission architecture trade option, we will also consider using an aeroshell to enable Saturn orbit insertion (SOI) via a Titan aerobraking maneuver (TAM), and then chemical propulsion techniques for the remainder of the mission. The aeroshell is deployed after SOI to reduce orbiter fuel mass for chemical propulsion maneuvers during the Enceladus phase of the mission and Titan orbit insertion (TOI). As another option, we will also consider retaining the aeroshell to put the orbiter in orbit around Titan. The aeroshell is considered a technology under development in the US, so cannot be considered as baseline at this time.

The orbiter is a key TandEM element, since it represents a significant increase in science return, but also serves as communication link between Earth and the balloon and probes/landers on the ground, while the Direct to Earth (DtE) communication possibility has to be assessed and can only be complementary. We will also take advantage of the low radiation environment, and reduce risk by miniaturizing the payload mass and power and thus cost. Structural mass reductions using new composite materials being developed independently by industry will be surveyed during the proposal period. Deployable high gain antenna (HGA) options will be considered as another mass reduction approach.

The baseline orbiter sub-system package includes dry spacecraft mass and $\Delta V \sim 3,000 \mathrm{~m} / \mathrm{s}$ chemical propulsion fuel mass. The launch mass is $\sim 2,586 \mathrm{~kg}$. 
The mass of a solar electric propulsion (SEP) module would be $1,080 \mathrm{~kg}$ for $18.7 \mathrm{~kW}$ of power (at $1 \mathrm{AU}$ ). The numbers are based on present mass, volume and power numbers for typical spacecraft and science instrument (SI) estimates and we expect with focused and independent technology developments these numbers will come down. Margins $\sim 20-30 \%$ have been added. Smaller margins $\sim 10 \%$ are used for fuel allocations. The data does show that by using the aerobraking option to put the orbiter in orbit around Saturn, with a launch mass of $\sim 1,653 \mathrm{~kg}$ one can use the Atlas V-521 launcher for the orbiter payload and as we discuss below can use a separate Atlas V-521 launcher for the Titan balloon, probes and carrier. For the baseline and the aerobraking option one can make ROM cost estimates of orbiter and mission operations (MO) during the study phase. Clearly, the most cost effective option is aerobraking so that our TDP will make the aeroshell technology a critical element for development.

\subsection{Titan Montgolfière}

Among different types of balloons, the most appropriate for Titan's case appears to be the Montgolfière - or hot air balloon-heated by the excess heat of two RTGs (Radioisotope Thermoelectric Generators).

The Titan Montgolfière has been studied by the Balloon Department of the CNES Toulouse Center which possesses an extensive knowledge of such vehicles as proved by the successful program called MIR (Montgolfière Infra Rouge) launched on a routine basis in the Earth's atmosphere since 1980. The studies show that the atmosphere of Titan appears as the most favourable of all the planetary atmospheres for balloon (especially Montgolfière) flight, because it is rather dense, since mainly composed of nitrogen with a surface pressure of $1.5 \mathrm{bar}$, and very cold (less than $100 \mathrm{~K}$ in the $0-70 \mathrm{~km}$ altitude range).

Compared to MIR on Earth, with the same temperature ratio and for the same lift, the balloon volume can be reduced because of a larger pressure on Titan ( $\sim 1$ bar) with respect to Earth ( $\sim 0.07$ bar $)$ at float altitude.

An orbiter is required as a data relay to Earth with a transmission rate of $20 \mathrm{kbs}^{-1}$ between the orbiter and the buoyant station(s) and two MultiMission Radioisotope Thermoelectric Generators (MMRTGs) are needed to produce a power of about $3.5 \mathrm{~kW}$ after 8 years of planetary cruise and assumed 2 years of Earth storage. In this case, the sustentation is provided by Titan's gas, filling the balloon and heated by an external energy source. It is therefore not necessary to carry the heavy tanks, whose mass create a major constraint on the balloon mission.

The drawback of the Montgolfière is that the difference in density between the aerostat gas and the ambient atmosphere is so small that a large volume is required. At Titan, a Montgolfière requires $1 \%$ of the heat required by Earth Montgolfières due to a combination of reduced cryogenic radiation, reduced heat transfer, and buoyancy inversely proportional to the square of 
the absolute temperature. Solar or IR atmospheric heating is too weak on Titan to be considered, catalytic decomposition of hydrazine and RTG heating are possibilities (preferably the latter).

The principle of a flight in Titan's atmosphere is to use the local gas heated by the excess thermal flux of the RTG system, which is baselined for providing electrical power to the balloon payload. The thermal effects coming from the Sun on the planet's atmosphere are negligible. The major question in the analysis of the flight is the convective exchanges with ambient; this has been treated by CNES with the help of the model of convection well validated by the MIR programme.

The results have been applied to the example of a $1,000 \mathrm{~m}^{3}$ balloon (Fig. 6).

With the MMRTGs as a heat source, the heat balance leads to a temperature difference of $3^{\circ} \mathrm{C}$ to $4^{\circ} \mathrm{C}$ providing sufficient lift for a typical payload mass.

The balloon would have a radius of $6.5 \mathrm{~m}$, a mass of $28 \mathrm{~kg}$ for a skin thickness of 30, $5 \mu \mathrm{m}$ made of a film of Kapton coated by FEP on both sides. This materiel is assembled by thermosoldering. Because of the expected instability of the vehicle in the temperature gradient, it is necessary to add a small $2.3 \mathrm{~m}$ radius balloon (mass $4 \mathrm{~kg}$ ) filled with hydrogen $(11 \mathrm{~kg})$.

The system would be deployed starting around $30 \mathrm{~km}$ of altitude and the balloons inflated while descending down to $3 \mathrm{~km}$ where the tanks and inflation system $(60 \mathrm{~kg})$ used to carry hydrogen would be jettisoned. Then the vehicle would climb back to a nominal float altitude of $10 \mathrm{~km}$ (Fig. 7). A valve placed

Fig. 6 Titan Montgolfière system description

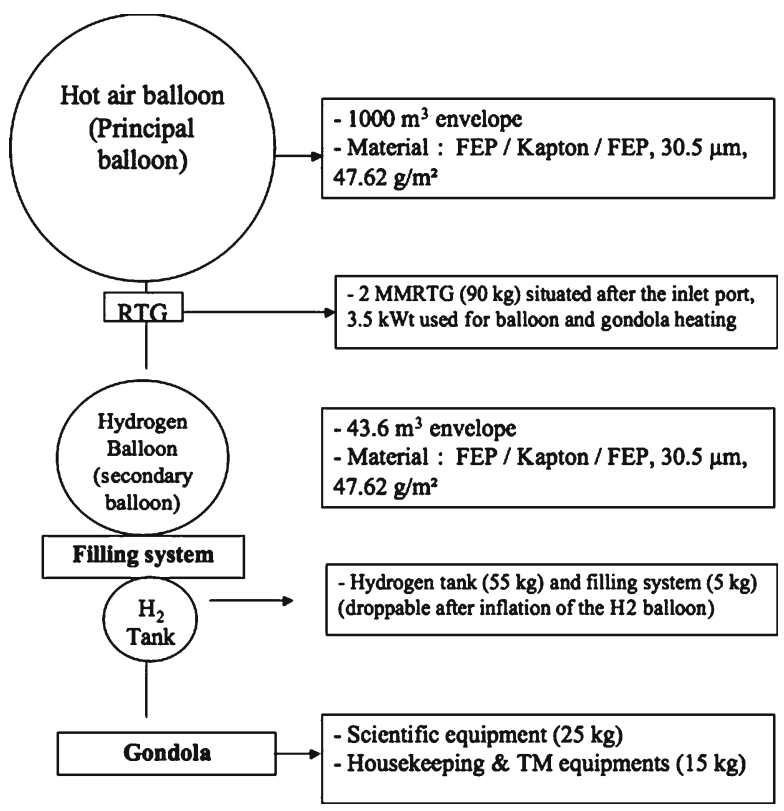




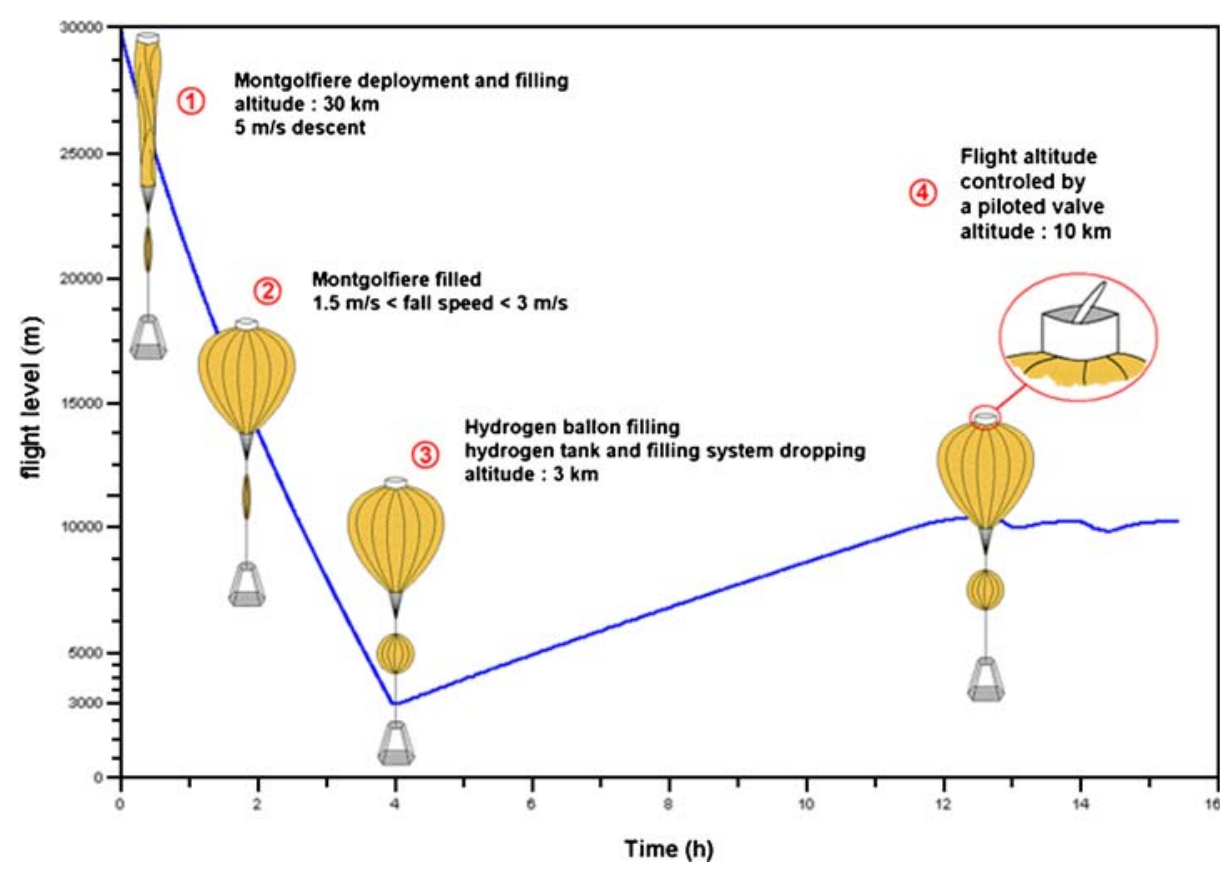

Fig. 7 Titan deployment and flight simulation

on top of the Montgolfière (Fig. 7) would be used for emptying partially the main balloon in order to obtain excursions in altitude (up to $25-30 \mathrm{~km}$ ).

Nominal ceiling could be achieved around $10 \mathrm{~km}$ by using the active control of the valve (mass of control system $=10 \mathrm{~kg}$ ). Another possibility is a ceiling around $3 \mathrm{~km}$. Complete filling corresponds to an infinite flight around $30 \mathrm{~km}$.

The total mass breakdown is $263 \mathrm{~kg}$ including $30 \mathrm{~kg}$ of margin. The mass available below the balloon is $130 \mathrm{~kg}$, including $90 \mathrm{~kg}$ of the RTGs; a gondola for housekeeping, data telecommunication and scientific instruments of $40 \mathrm{~kg}$.

It is estimated that a scientific payload of at least $20 \mathrm{~kg}$ would be available out of the $40 \mathrm{~kg}$ gondola.

The lifetime is anticipated to be long (months to years) since leaks do not impair performances in a Montgolfière. However the lifetime would be reduced due to $\mathrm{H}_{2}$ leakage from the secondary balloon.

\subsection{Probes}

Probes provide in situ measurements not possible with orbiting or airborne instruments; ground truth; and support interpretation of orbiting instrument data with hard ground information. 
We propose a baseline of Titan descent and landing mini-probes, in line with the Huygens heritage, as well as micro-penetrators and lander probes for Enceladus to address the mission requirements in complementary ways. Their selection will ultimately depend on available mass, capability and risk.

\subsubsection{Titan mini-probes}

The Titan mini probe design will benefit from the Huygens probe experience, and from the GEP-ExoMars (Geophysics Package) development (itself originated from the NETLANDER project).

The arrival on Titan will be on very similar conditions to the Huygens one. The aeroshell and the parachute system will hence be derived from the Huygens one, downscaled to produce the same ballistic coefficient. An aeroshell diameter of $1.3 \mathrm{~m}$ will provide the same entry as the Huygens one.

The surface module design will be strongly inherited from the GEPExoMars, which has similar environment and mission constraints:

- Autonomy;

- Cold thermal environment;

- Low solar power availability.

The negligible solar flux on Titan's surface led to implement a radioisotopic device for electrical power generation. The GEP development of a European RTG could be used to satisfy the Titan mini-probes' power requirement. This RTG could be based on a Russian RHU (radioisotope heater unit) design, with a European thermoelectric generator (TEG) developed in Europe, which is expected to provide an increased system efficiency, compared to the RITEC TEG used on the (unsuccessful) MARS-96 mission. The electrical power will be in the order of $4 \mathrm{~W}$, which leads to a time-sharing payload management.

The power system will be based on the coupled RTG/secondary battery with an operational schedule taking into account science measurements campaign, telecommunication session to the Orbiter and battery charging.

The Titan mini-probes will benefit from electronic integrated design in order to reduce the mass, and to share the thermal dissipation to the overall probe elements. The RTG will be embedded within the overall equipments. The avionics will benefit from the ExoMars GEP development. The thermal control will inherit from the Huygens design which has demonstrated a very good behaviour. A high TRL level is applicable for the Entry and Descent system, thanks to the strong heritage from Huygens. A TRL level of eight is hence considered.

For the parachute system, a resizing is necessary considering the lower mass with regard to the Huygens probe. A TRL of eight is considered. The surface module will be strongly derived from the GEP-ExoMars module.

Considering the specific operational concept required by the electrical power management (time sharing between instruments and telecommunication), the data handling will necessitate specific development in terms of operation. A TRL level of seven could be considered for the data handling. 


\subsubsection{Micro-penetrators}

We propose low mass kinetic penetrators capable of withstanding impact at velocities up to several hundred $\mathrm{m} / \mathrm{s}$, and embed themselves under the surface to a depth of up to a few metres.

The whole system comprises three major elements: (a) carrier accommodation and ejection system; (b) a descent probe which contains a thruster and attitude control assembly, a possible descent camera; and also includes (c) the penetrator itself (if penetrators were also used for Titan, we could propose a largely common penetrator design and payload). Figure 8 shows a conceptual design of such a penetrator.

A preliminary mass estimate for such a common penetrator is $\sim 5 \mathrm{~kg}$, which includes a $\sim 1.7 \mathrm{~kg}$ core instrument suite comprising seismometers, geochemistry package, thermal sensors, accelerometers and descent camera (see Table 10). Complementary payload instruments (e.g. subsurface mineralogy/astrobiology camera, magnetometer, etc.) will be considered for inclusion after further study of science priorities, technical maturity, and available mass. The only payload item which may not be common to both Enceladus and Titan is that of the chemical sensor because of the atmospheric presence on Titan.

Though there is space qualification heritage for such systems with Lunar-A and Deep Space-2 (DS2) at impact velocities proposed here, together with extensive military experience with instrumented shells, some particular adaptions to this mission will be required e.g. (ruggedisation of new technology science instruments; possible effects on long cruise phase on thrusters (solid) fuel; low mass attitude control system; extending battery power system lifetime by employing RHU's; and possible need for a trailing aerial for communications through subsurface material).

Fig. 8 Penetrator descent module

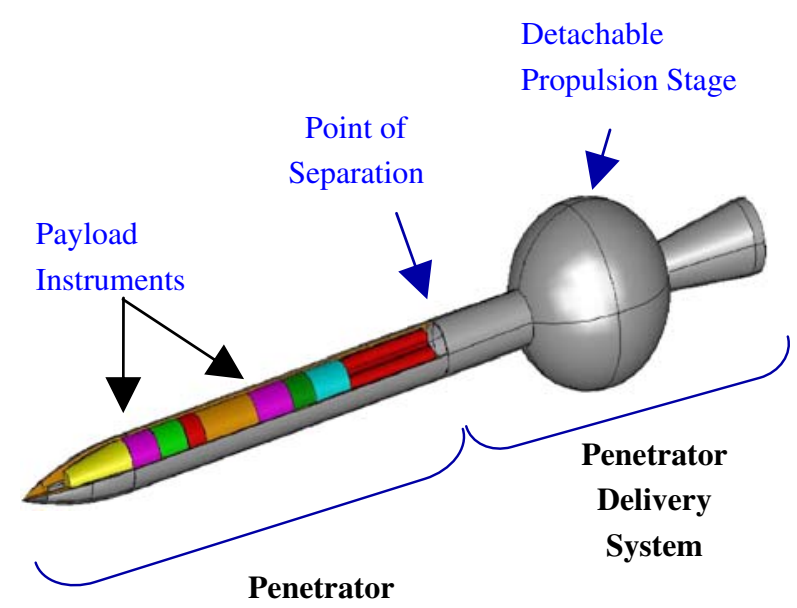


Table 10 Possible penetrator science payload

\begin{tabular}{lll}
\hline Penetrator payload instrument & Mass & Heritage \\
\hline Descent camera $(\mathrm{g})$ & 10 & Beagle2, ExoMars \\
Accelerometer and tiltmeter $(\mathrm{g})$ & 66 & DS2, Lunar-A \\
Geochemistry package $(\mathrm{g})$ & 260 & Beagle-2 XRS \\
Water-volatile experiment $(\mathrm{g})$ & 750 & DS2, Ptolemy (Rosetta) \\
Seismometer $(\mathrm{g})$ & 300 & ExoMars \\
Thermal/heat flow $(\mathrm{g})$ & 300 & Lunar-A \\
Permittivity $(\mathrm{g})$ & $\sim 100$ & IWF (Graz) \\
Mineralogy/astrobiology camera $(\mathrm{g})$ & $\sim 200$ & Ground based (USA) \\
Magnetometer $(\mathrm{g})$ & 60 & Various space missions \\
Total penetrator payload mass $(\mathrm{kg})$ & $\sim 2$ & \\
\hline
\end{tabular}

Enceladus penetrators A two penetrator system is proposed to allow investigation of two surface terrains and provide some fault tolerance. Deployment from the Titan-Enceladus orbiter requires a thruster to reduce the $\sim 3.8 \mathrm{~km} / \mathrm{s}$ $\Delta V$ to an impact velocity around $200-300 \mathrm{~m} / \mathrm{s}$. Preliminary calculations for solid fuel system (such as one employed for Lunar-A), with an ejection velocity of $\sim 2,600 \mathrm{~m} / \mathrm{s}$, equates to a thruster system comprising $\sim 26.8 \mathrm{~kg}$ fuel $+2.5 \mathrm{~kg}$ for associated tanks, structure and motor. Use of a bi-propellant system, could result in a slightly lower separation mass and provide additional control. The result is a total separation mass for a two penetrator system estimated at around $77 \mathrm{~kg}$. Thus, a nominally allocated $100 \mathrm{~kg}$ for this system provides a $23 \mathrm{~kg}$ contingency which could be used to enable additional complementary payload instruments if sufficient mass is available.

Titan penetrators These may be deployed either from orbit or from the hot air balloon. In either case, descent will be significantly affected by Titan's thick atmosphere. This may allow fin-based ACS which have commonly been used by the defence sector on Earth. Here, thermal insulation from the cold landed phase may also be useful for protection against atmospheric heating. Communications could be to the orbiter or balloon, depending on balloon location with time. The descent probe subsystems will be largely identical with those for Enceladus except for the ACS to reduce costs.

Orbit deployment Allows deployment of multiple penetrators to pre-selected different regions/terrains, at closely spaced times to also allow a simultaneous small seismic network to be operational. There is a heritage of direct atmospheric entry with the Titan Huygens probe. A preliminary analysis using comparison with Huygens indicates a mass per descent probe of around 12 to $30 \mathrm{~kg}$ respectively, which is also in agreement with simple use of thrusters and ACS system to ensure correct entry orientation and sufficient penetration.

Balloon deployment This allows low altitude and perhaps lower mass deployment, but targeting will depend upon the vagaries of Titan's winds, and consequent ability to ensure a contemporary seismic network is unlikely if 
specific terrain targeting is a priority. A mass estimate for a thruster based balloon deployment is $\sim 8.5 \mathrm{~kg} /$ descent probe, based on a reduced $1.5 \mathrm{~kg}$ probe accommodation and ejection system, and $\sim 1.0 \mathrm{~kg}$ thruster mass.

\subsection{Key technology areas}

This future ambitious program will require ESA and its international partners to investigate innovative and critical technologies, such as: consolidating and improving upon the Huygens entry technology and extending it to controlled dips in the atmosphere which are required for aerocapture; the orbital stability of Titan's aerocaptured orbiter; technology development for the balloons the mini-probes and the penetrators; DtE communications, etc.

A technology implementation plan, for infusing these and other technologies into the TandEM mission, will be developed during the pre-phase A study. The list of study tasks also includes:

1. RTG heat exchanger, material development (two-layer balloon concept) and drop and deployment test for Montgolfière balloon. Small RTGs enable many new options e.g. long-lived seismic stations).

2. Microelectronics development which can be done under low radiation specification for mission.

3. Develop tether system and surface-liquid sampling capabilities.

4. Trade studies on solar electric propulsion.

5. Trajectory designs for probe/landers/ penetrators releases on Titan and Enceladus.

6. On-board science autonomy: data selection, compression and storage $\mathrm{C} \& \mathrm{DH}$ and Telecom systems.

\section{Communications and outreach}

Astronomy plays an important role in every day life at all latitudes and longitudes and even the layperson is very much interested, as perhaps it seems that this science is imprinted in our genes and we have an atavistic interest for it. It is recognised in this proposal that communication and outreach is an important part of this mission. The purpose is the rapid dissemination of information to scientists as well as information to the general public. Titan and Enceladus are complex systems with many interacting components like the Earth.

The interest of the informed and layman public in the Saturnian system has steadily increased in the past, with a peak in the current Cassini-Huygens era. Images from the Saturnian system have regularly been featured as the Astronomy Picture of the Day and have attracted international media broadcasting. This can only be amplified by a return to Saturn's neighbourhood. TandEM will be appealing for educational institutions at different levels. 
There are several points about the TandEM mission that make it unique and of high interest to the general public. Titan's being another world with many similarities to an early Earth is one. Although it is a moon, it is larger than Mercury, has an active geology and a significant atmosphere. The geology has Earth-like scientific and educational analogues despite its alien environment: Mountains of ice, lakes of methane/ethane, dunes where the dust is ice or even some organic material. The atmosphere is probably similar in composition and density to that of the early Earth's. Although Titan is extremely cold, there are complex organic chemical reactions occurring in the atmosphere, which may be similar to those that occurred on Earth.

The TandEM mission makes full use of Titan's atmosphere for aerobraking/aero-capture and to enable a highly mobile instrument platform, i.e. a hot air balloon. The physics of a hot air balloon demonstrate practical aspects of physics used in school curricula e.g. mass, density and the ideal gas laws. There are many websites describing design and construction of model hot air balloons, showing that there is a general interest in hot air balloons.

During pre-launch and cruise phases there can be competitions to name and design logos for the balloon and probes/landers for Titan and Enceladus (similar to the Philae lander for the Rosetta mission). Gradually building media awareness through briefings and pres releases. Design educational programs. Enable people to add their names on a CD that will be carried on the gondola.

During the Titan/Enceladus encounter: Dissemination of information on web. News alerts to subscribers on mobile phones by text messaging. Enabling the general public to help choose where to land the probes and penetrators and capture balloon samples.

Public outreach activities will include: internet sites, leaflets, a comic, 3D constructions with cardboard or plastic, public lectures, TV and radio programs, CD and DVDs, exhibitions, articles in magazines and newspapers, theatrical plays for Saturn and its satellites with emphasis on Titan and Enceladus. The supports could be produced and distributed by ESA and all its partners to media and newspapers and possibly through newspapers to the general public.

Interactive exhibitions are considered to spread around European institutions and planetariums with the collaboration of the local astronomy and physicists societies. their main subject will be a virtual tour, beginning with the evolution of Astronomy from the ancient years including the famous Antikythera mechanism, the oldest astronomical computer, and ending with a travel to the Saturniann system with final destinations Titan and Enceladus. After having watched the show, children will be involved at a game, which concern the design of a mission to Titan and Enceladus and a balloon trip to fly over and study it.

Moreover, several contests will be organized by ESA among the European schools, in order to collaborate each other and create mission posters, log and flag. An expedition to name the TandEM instrumentation could be also held. Ideas and resources will naturally flow in regard to such a mission. 


\section{Conclusions and recommendations}

The TandEM mission, currently under study by ESA and its international partners will make an enormous impact on the planetary science community. Indeed, the list of questions pertaining to Titan as a system is lengthy as befits a world perhaps second only to Earth in its level of geologic and atmospheric activity (as described in Section 3.1). The origin and evolution of the puzzling moon, Enceladus, is also a critical end point for understanding the formation of planetary systems and its astrobiological potential, as explained in Section 3.2. In addition, the variability of the magnetospheric plasma, neutral gas, Ering ice grain density, radio emissions, and co-rotation of Saturn's planetary magnetic field in response to Enceladus' plume activity need to be addressed to understand the present Saturnian system of satellites, rings and plasma.

Such a mission will also certainly stimulate important technological advances through the challenging new components required for this investigation. In particular, we have begun to develop conceptual designs for delivering the science payload included in the orbiters, aerial platforms and probes, and to define a launch/delivery/communication management architecture. The TandEM mission concept will allow us to identify key areas for technology development and corresponding development of a technology plan.

It is highly recommended if not mandatory for the international space agencies to combine efforts and collaborate in order to make such an ambitious endeavour a reality. ESA and its partners will share the mission operations according to a plan to be defined during the study phases. TandEM will require new technologies and capabilities so that the science goals can be achieved within a cost cap defined with acceptable risk for a class-L mission (similar to the one of Cassini-Huygens). The cost target considered here is widely recognized in all studies for a return to Titan and Enceladus to be reasonable given the cost of the highest priority science. International participation among ESA, NASA and other potential partners will play a key role in achieving all the science goals of this mission, which will revolutionize our understanding not only of the Saturnian system, but of the entire Solar system.

Acknowledgements We want to thank Ivan Juiz who first put together the TandEM web site, and our current webmaster: Renaud Romagnan. We are also grateful to Sylvain Cnudde for considerable artistic work on TandEM, and to SIGAL@LESIA.

\section{References}

1. Alibert, Y., Mousis, O.: Astron. Astrophys. 465, 1051-1060 (2007)

2. Atreya, S.: Science 316, 843-845 (2007)

3. Atreya, S.K.: Atmospheres and ionospheres of the outer planets and their satellites, pp. 183186. Springer, New York (1986)

4. Atreya, S.K., et al.: Science 201, 611-613 (1978)

5. Atreya, S.K., et al.: Planet. Space Sci. 54, 1177-1187 (2006)

6. Backes, H., et al.: Science 308, 992-995 (2005)

7. Coates, A.J., et al.: Geophys. Res. Lett. 34, L22103 (2007) 
8. Coustenis, A., et al.: Icarus 189, 35-62 (2007)

9. Dougherty, M., et al.: Science 311, 1406 (2006)

10. Engel, S., Lunine, J.I., Norton, D.L.: J. Geophys. Res. 99(E2), 3745-3752 (1994)

11. Fortes, A.D.: Icarus 146, 444-452 (2000)

12. Fortes, A.D., et al.: Icarus 188, 139-153 (2007)

13. Griffith, C., et al.: Science 310, 474-477 (2005)

14. Grott, M., et al.: Icarus 191, 203-210 (2007)

15. Hersant, F., et al.: Planet. Space Sci. 52, 623-641 (2004)

16. Hersant, F., et al.: Icarus (2007, in press)

17. Hurford, T.A., et al.: Nature 447, 292-294 (2007)

18. Israël, G., et al.: Nature 438, 796-799 (2005)

19. Khare, B.N., et al.: Icarus 68, 176-184 (1986)

20. Kieffer, S.W., et al.: Science 314, 1764 (2006)

21. Lorenz, R.D., et al.: Geophys. Res. Lett. 34, L07204 (2007)

22. Magni, G., Coradini, A.: Planet. Space Sci. 52, 343-360 (2004)

23. Matson, D.L., et al.: Icarus 187, 569-573 (2007)

24. McKay, C.P., et al.: Icarus 80, 23-53 (1989)

25. McKay, C.P., et al.: Science 253, 1118-1121 (1991)

26. McKay, C.P., Smith, H.D.: Icarus 178, 274-276 (2005)

27. Niemann, H.B., et al.: Nature 438, 779-784 (2005)

28. Nguyen, M.-J., et al.: Planet. Space Sci. 55, 2010-2014 (2007)

29. Nimmo, F., et al.: Nature 447, 289-291 (2007)

30. Owen, T., et al.: Phys. Uspekhi. 48, 635-639 (2005)

31. Owen, T., et al.: Faraday Discuss. 133, 387-391 (2006)

32. Palguta, J., et al.: Icarus 180, 428-441 (2006)

33. Porco, C.C., et al.: Science 311, 1393-1401 (2006)

34. Rappaport, N., et al.: Icarus 126, 313-323 (1997)

35. Rappaport, N., et al.: Icarus 190, 175-178 (2007)

36. Raulin, F.: Space Sci. Rev. Available at http://www.springerlink.com/content/ 0527749877335772/. (2007, in press)

37. Schubert, G., et al.: Icarus 188, 345-355 (2007)

38. Soderblom, L.A., et al.: Planet. Space Sci. 55, 2025-2036 (2007a)

39. Soderblom, L.A., et al.: Planet. Space Sci. 55, 2015-2024 (2007b)

40. Sohl, F., et al.: J. Geophys. Res. 108, 5130 (2003). doi:10.1029/2003JE002044

41. Sotin, C.: Nature 445, 29 (2007)

42. Sotin, C., et al.: Nature 435, 786-789 (2005)

43. Spencer, J.R., et al.: Science 311, 1401-1405 (2006)

44. Spitale, J.N., Porco, C.C.: Nature 449, 695-697 (2007)

45. Tian, F., et al.: Science 308, 1014-1017 (2005)

46. Tobie, G., et al.: Icarus 175, 496-502 (2005)

47. Tobie, G., et al.: Nature 440, 61-64 (2006)

48. Tobie, G., et al.: Icarus (2007, in press)

49. Tokano, T., et al.: Nature 442, 432-435 (2006)

50. Waite, H., et al.: Science 311, 1419-1422 (2006)

51. Waite, H., et al.: Science 316, 870-875 (2007) 